\title{
QUADRATIC FLOW PAST AXIALLY SYMMETRIC BODIES AT LOW REYNOLDS NUMBER: A NEW APPROACH
}

\author{
Deepak Kumar Srivastava, Nirmal Srivastava \\ Department of Mathematics, B.S.N.V. Post Graduate College(University of Lucknow) \\ Station Road, Charbagh, Lucknow(U.P.)-226001, India \\ dksflow@hotmail.com, nirmalsri.25@gmail.com \\ RAJA RAM YADAV \\ Department of Mathematics, University of Lucknow, Lucknow(U.P.)-226007, India \\ yadav_rr2@yahoo.co.in
}

[Received: September 27, 2012]

\begin{abstract}
In this paper, a new approach to tackle the quadratic flow past axially symmetric bodies at low Reynolds number is presented. Firstly, a new method for the evaluation of drag based on DS conjecture (Datta and Srivastava, [1, 1999]) and surface average velocity defined by Chwang and $\mathrm{Wu}$ (part 2 and 3 [2, 3, 1975]) on a body placed in quadratic flows like parabolic and stagnation like parabolic is presented. Later on, these general values of drag are utilized for bodies like sphere, spheroid and egg-shaped in both axial and transverse situations. Author claims that the proposed method is easy and very handy to study the quadratic flow past not only for a isolated body but also for assemblage of bodies situated along the axis of symmetry in comparison to complicated methods like distribution of basic singularities which is not applicable to all axially symmetric bodies except sphere and spheroidal bodies. Numerical values of drag in every flow situation have been calculated and presented in Tables 1-9. The respective variations with respect to eccentricity ' $\mathrm{e}$ ' are depicted via figures. Limiting cases of slender spheroid and flat circular disk are also discussed.
\end{abstract}

Mathematical Subject Classification: 76D07

Keywords: Quadratic flows, parabolic flow, stagnation like parabolic flow, Stokes drag

\section{INTRODUCTION}

In physical and biological science, and in engineering, there is a wide range of problems of interest like sedimentation problem, lubrication processes etc. concerning the flow of a viscous fluid in which a solitary or a large number of bodies of microscopic scale are moving, either being carried about passively by the flow, such as solid particles in sedimentation, or moving actively as in the locomotion of micro-organisms. In the case of suspensions containing small particles, the presence of the particles will influence the bulk properties of the suspension, which is a subject of general interest in Rheology. In the motion of micro-organisms, the propulsion velocity depends critically on their body shapes and modes of motion, as evidenced in the flagellar and 
cilliary movements and their variations. A common feature of these flow phenomena is that the motion of the small objects relative to the surrounding fluid has a small characteristic Reynolds number Re. Typical values of Re may range from order unity, for sand particles settling in water, for example, down to $10^{-2}$ to $10^{-6}$, for various micro-organisms. In this low range of Reynolds numbers, the inertia of the surrounding fluid becomes insignificant compared with viscous effects and is generally neglected and the Navier-Stokes equations of motion reduce to the Stokes equations as a first approximation. The zero Reynolds number flow is called Stokes flow. The hydromechanics of low Reynolds number flows play an important role in the study of rheology, lubrication theory, micro-organism locomotion and many areas of biophysical and geophysical interest. In the case when the inertial effects are negligible compared with the viscous forces, the Navier-stokes equations are usually simplified to Stokes equations as a first approximation. Determination of the solutions for the Stokes flows, however, isstill recognized to be difficult in general for arbitrary body shapes. As a consequence, not many exact solutions are known. Of the few analytical methods available for solving Stokes flow problems, one is the boundary value method, which is based on the choice of an appropriate co-ordinate system to facilitate separation of the variables for the body geometry under consideration. Another is the singularity method, whose accuracy depends largely on whether the correct types of singularity are used and how their spatial distributions are chosen. The boundary value method seems to have been widely adopted in practice, more so than the singularity method. Stokes flow of an arbitrary body is of interest in biological phenomena and chemical engineering. In fact, the body with simple form such as sphere or ellipsoid is less encountered in practice. The body, which is presented in science and technology, often takes a complex arbitrary form. For example, under normal condition, the erythrocyte is a biconcave disk in shape, which can easily change its form and present different contour in blood motion due to its deformability. In second half of twentieth century, a considerable progress has been made in treating the Stokes flow of an arbitrary body.

An exact solution for the motion of a spheroidal particle placed in a quadratic as well as in a linear flow of incompressible viscous fluid is very useful in the study of blood flow and general suspension rheology. In particular, a correct description of the behavior of a spheroidal particle in a paraboloidal or Poiseuille flow will facilitate accurate calculation of the bulk flow properties of tube flows of dilute or concentrated suspensions of blood cells, long-chain polymers or any other biological supra-macromolecules. When the Reynolds number based on the particle size, the local flow velocity and the kinematic viscosity of the surrounding fluid is very small, as in the case of microcirculation of blood cells, the inertial effects of the fluid can be neglected and the Navier-Stokes equations of motion reduce to the Stokes equations as a first approximation.

All these motions are characterized by low Reynolds numbers and are described by the solution of the Stokes equations. Although the Stokes equations are linear, to obtain exact solutions to them for arbitrary body shapes or complicated flow conditions is still a formidable task. There are only relatively few problems in which it is possible 
to solve exactly the creeping motion equations for flow around a single isolated solid body. Stokes [4, 1851] calculated the flow around a solid sphere undergoing uniform translation through a viscous fluid whilst Oberbeck [5, 1876] solved the problem in which an ellipsoid translates through liquid at a constant speed in an arbitrary direction. Edwards [6, 1892], applying the same technique, obtained the solution for the steady motion of a viscous fluid in which an ellipsoid is constrained to rotate about a principal axis. The motion of an ellipsoidal particle in a general linear flow of viscous fluid at low Reynolds number has been solved by Jeffery [7, 1922], whose solution was also built up using ellipsoidal harmonics. The analysis described by Jeffery extended further by Taylor [8, 1923]. Goldstein [9, 1929] obtained a force on a solid body moving through viscous fluid. Lighthill [10, 1952] studied the problem of squirming motion of nearly spherical deformable bodies through liquids at very small Reynolds number. Hill and Power [11, 1956] have obtained arbitrarily closed approximations of drag by proving a complimentary pair of extremum principles for a Newtonian viscous fluid in quasi-static flow.

In a series of studies over low-Reynolds-number-flow, Chwang and $\mathrm{Wu}$ [12, part 1, 1974] have developed an effective method of solution for arbitrary body shapes. In this first part authors have considered the viscous flow generated by pure rotation of an axisymmetric body having an arbitrary prolate form, the inertia forces being assumed to have a negligible effect on the flow. The method of solution explored in this paper is based on a spatial distribution of singular torques, called rotlets, by which the rotational motion of a given body can be represented. Exact expressions of torque are determined in closed form for a number of body shapes, including the dumbbell profile, elongated rods and some prolate forms. Chwang and $\mathrm{Wu}$ 2, part 2, 1975] explored the fundamental singular solutions for Stokes flow that could be useful for constructing solutions over a wide range of free-stream profiles and body shapes. Authors have employed these fundamental singularities (Stokeslet and their derivatives like rotlets, stresslets, potential doublets and higher orderpoles) to construct exact solutions to a number of exterior and interior Stokes-flow problems for several specific body shapes translating and rotating in a viscous fluid which may itself be providing a primary flow. The different primary flows considered here include the uniform stream, shear flows, parabolic profiles and extensional flows(hyperbolic profiles), while the body shapes cover prolate spheroids, spheres and circular cylinders. Chwang [3, part 3, 1975] has been found exact solutions in closed form (expressions of drag) using singularity method for various quadratic flows of an unbounded incompressible viscous fluid at low Reynolds numbers past a prolate spheroid with an arbitrary orientation with respect to the fluid. The quadratic flows considered here include unidirectional paraboloidal flows, with either an elliptic or a hyperbolic velocity distribution, and stagnation-like quadratic flows as typical representations. Chwang and $\mathrm{Wu}[13$, part 4, 1975] have analyzed the problem of a uniform transverse flow past a prolate spheroid of arbitrary aspect ratio at low Reynolds numbers by the method of matched asymptotic expansions. They have found expressions of drag depending on two Reynolds numbers, one based on the semi-minor axis $b, R_{b}=U b / \nu$, and the other on the semi-major axis $a, R_{a}=U a / \nu$. Ho and Leal [15, 1976] considered the 
migration of a rigid sphere in a two-dimensional unidirectional shear flow of a second order fluid. Chan and Leal [31, 1977] wrote a note on the motion of a spherical particle in a general quadratic flow of a second order fluid. Parker [16, 1977] considered the two-dimensional motion of a projectile experiencing a constant gravitational force and a fluid drag force which is quadratic in the projectiles speed. Johnson and Wu [14, part 5, 1979] elucidated the characteristics of the general stokes flow for slender bodies of finite centre-line curvature. The singularity method for Stokes flow has been employed by authors to construct the solutions to the flow past a slender torus.

Majhi and Vasudevaiah [17, 1982] considered the axisymmetric parabolic shear flow past a spinning sphere in an unbounded viscous medium using the matching technique and taking the non-uniform shear as the dominant feature, where the Reynolds number based on parabolic shear $R_{e} \leq 1$ and rotational Reynolds number based on the angular rotation of sphere $R_{o} \leq 1$ are such that $R_{o}^{2} / R_{e}=O(1)$. Kaloni [18, 1983] studied the motion of a rigid sphere, suspended in micropolar fluid which is undergoing a slow unidirectional two-dimensional flow. Keh and Anderson [19, 1984] obtained the configurational distribution function of dumbbell macromolecules(rigid and linearly elastic) in a quadratic rectilinear flow. Yuan and $\mathrm{Wu}$ [20, 1987] obtained the analytic expressions in closed form for flow field by distributing continuously the image Sampsonlets with respect to the plane and by applying the constant density, linear and the parabolic approximation. Yang and Hong [21, 1988] found exact solutions in closed form using the eigen function-expansion method for various linear and quadratic flows of an unbounded incompressible viscous liquid at low Reynolds number past a porous sphere with a uniform permeability distribution. Seki [22, 1996] studied the motion of a rigid ellipsoidal particle freely suspended in a Poiseuille flow(parabolic flow) of an incompressible Newtonian fluid through a narrow tube numerically in the zero-Reynolds-number limit. Haber and Brenner [23, 24, 1984, 1999] investigated analytically the quasi-steady hydrodynamic Stokes drag force and torque exerted on each of $\mathrm{N}$ non-identical particles immersed in a general quadratic undisturbed flow field at infinity. Datta \& Srivastava [1, 1999] advanced a new approach to evaluate the drag force in a simple way on a restricted axially symmetric body placed in a uniform stream (i) parallel to its axis, (ii) transverse to its axis, when the flow is governed by the Stokes equations under no-slip boundary conditions. Authors have evaluated the analytic closed form expressions of drag for spheroids, deformed sphere, cycloidal body, an egg-shaped body. Palaniappan and Daripa [25, 2000] have found exact analytical solutions for the steady state creeping flow in and around a vapor-liquid compound droplet, consisting of two orthogonally intersecting spheres of arbitrary radii(a and b), submerged in axisymmetric extensional(hyperbolic) and paraboloidal flows of fluid with viscosity $\mu$. Lin et al. [26, 2005] studied the hydrodynamic interaction between two neutrally-buoyant smooth spheres moving at negligible Reynolds numbers in an unbounded plane Poiseuille flow calculated by three-dimensional boundary element (BEM) simulations. Pasol et al. [27, 2006] provided comprehensive results for the creeping flow around a spherical particle in a viscous fluid close to a plane wall, when the external velocity is parallel to the wall and varies as a second degree polynomial in the coordinates. By using bipolar coordinates technique, authors have 
concluded that by linearity of Stokes equations, the solution is a sum of flows for typical unperturbed flows: a pure shear flow, a 'modulated shear flow', for which the rate of shear varies linearly in the direction normal to the wall, and a quadratic flow. Prakash et al. [28, 2012] studied the hydrodynamics of a porous sphere in an oscillatory viscous flow of an incompressible Newtonian fluid. Authors have derived the Faxen's law for drag and torque acting on the surface of the porous sphere. In the later part of the paper, examples of uniform flow, oscillating Stokeslets, oscillatory shear flow and quadratic shear flow are discussed.

In the present paper, author tried to advanced the own conjecture (Datta \& Srivastava [1, 1999]) to evaluate the closed form analytic expressions of drag over axisymmetric bodies placed under different primary flows including uniform stream, parabolic profiles and stagnation like parabolic profiles by considering the surface average of the primary flow velocity. This conjecture is briefly explained in Section 2. Reader is advisable to go through the author's (Srivastava et al., [29, 2012]) recently published paper for complete detail regarding its application to class of oblate bodies.

\section{BODY GEOMETRY AND METHOD}

2.1. Geometry of the body. Let us consider the axially symmetric body of characteristic length $L$ placed along its axis (x-axis, say) in a uniform stream $U$ of viscous fluid of density $\rho_{1}$ and kinematic viscosity $\nu$. When the Reynolds number $U L / \nu$ is small, the motion is governed by the Stokes equations (Happel and Brenner, 30, 1964]),

$$
\mathbf{0}=-\left(\frac{1}{\rho_{1}}\right) \operatorname{grad} p+\nu \nabla^{2} u, \quad \operatorname{div} \mathbf{u}=0
$$

subject to the no-slip boundary condition.

We have taken up the class of those axially symmetric bodies which possesses continuously turning tangent, placed in a uniform stream $U$ along the axis of symmetry (which is X-axis), as well as constant radius $b$ of maximum circular cross-section at the mid of the body. This axi-symmetric body is obtained by the revolution of meridional plane curve (depicted in Figure 1) about axis of symmetry which obeys the following limitations:

i. Tangents at the points $\mathrm{A}$, on the $\mathrm{x}$-axis , must be vertical,

ii. Tangents at the points $\mathrm{B}$, on the $\mathrm{y}$-axis, must be horizontal,

iii. The semi-transverse axis length ' $b$ ' must be fixed.

The point $\mathrm{P}$ on the curve is may be represented by the Cartesian coordinates $(x, y)$ or polar coordinates $(r, \theta)$ respectively, $\mathrm{PN}$ and $\mathrm{PM}$ are the length of tangent and normal at the point $\mathrm{P}$. The symbol $R$ stands for the intercepting length of normal between the point on the curve and point on axis of symmetry and symbol $\alpha$ is the slope of normal PM which can be vary from 0 to $\pi$. 


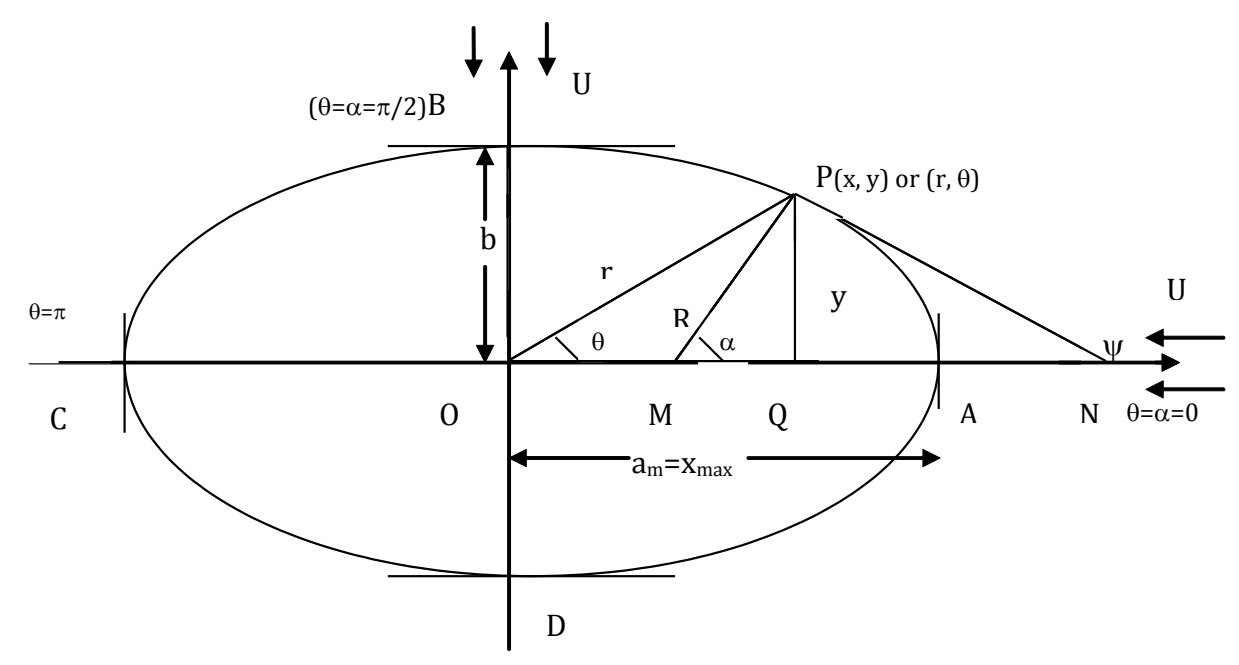

Figure 1. Geometry of the axially symmetric body

2.2. Axial flow. The expression of Stokes drag on such type of axially symmetric bodies placed in axial flow(uniform flow parallel to the axis of symmetry) is given by (Datta \& Srivastava [1, 1999])

$$
F_{\|}=\frac{1}{2} \frac{\lambda b^{2}}{h_{\|}}, \quad \text { where } \quad \lambda=6 \pi \mu U_{\|}
$$

and

$$
h_{\|}=\left(\frac{3}{8}\right) \int_{0}^{\pi} R \sin ^{3} \alpha \mathrm{d} \alpha .
$$

where the suffix $\|$ has been introduced to assert that the force is in the axial direction.

Sometimes it will be convenient to work in Cartesian co-ordinates. Therefore, referring to Figure 1 for the profile geometry, we have

$$
y=R \sin \alpha, \quad \tan \alpha=-\left(\frac{\mathrm{d} y}{\mathrm{~d} x}\right)^{-1}=-\frac{\mathrm{d} x}{\mathrm{~d} y}=-x^{\prime} .
$$

Using above transformation, we may express 2.3 as

$$
h_{\|}=-\frac{3}{4} \int_{0}^{a} \frac{y y^{\prime \prime}}{\left(1+\left(y^{\prime}\right)^{2}\right)^{2}} \mathrm{~d} x
$$

where $2 a_{m}$ represents the axial length of the body and dashes represents derivatives with respect to $x$. In the sequel, it will be found simpler to work with $y$ as the independent variable. Thus, $h_{\|}$assumes the form

$$
h_{\|}=-\frac{3}{4} \int_{0}^{b} \frac{y\left(x^{\prime}\right)^{2} x^{\prime \prime}}{\left(1+\left(x^{\prime}\right)^{2}\right)^{2}} \mathrm{~d} y
$$


where dashes represents derivatives with respect to $y$.

2.3. Transverse flow. The expression of Stokes drag on such type of axially symmetric bodies placed in transverse flow (uniform flow perpendicular to the axis of symmetry) is given by (Datta \& Srivastava [1, 1999])

$$
F_{\perp}=\left(\frac{1}{2}\right) \frac{\lambda b^{2}}{h_{y}} \quad \text { where } \quad \lambda=6 \pi \mu U_{\perp},
$$

and

$$
h_{\perp}=\left(\frac{3}{16}\right) \int_{0}^{\pi} R\left(2 \sin \alpha-\sin ^{3} \alpha\right) \mathrm{d} \alpha .
$$

According to the same manner as we did in axial flow equation (2.8) may also be written in Cartesian form as (in both cases having $x$ and $y$ treated as independent)

$$
h_{\perp}=-\frac{3}{8} \int_{0}^{a} \frac{y y^{\prime \prime}\left[1+2\left(y^{\prime}\right)^{2}\right]}{\left[1+\left(y^{\prime}\right)^{2}\right]^{2}} \mathrm{~d} x
$$

and

$$
h_{\perp}=-\frac{3}{8} \int_{0}^{b} \frac{y x^{\prime \prime}\left[2+\left(x^{\prime}\right)^{2}\right]}{\left[1+\left(x^{\prime}\right)^{2}\right]^{2}} \mathrm{~d} y
$$

In (2.9) and 2.1) the dashes represents derivatives with respect to $x$ and $y$ respectively. The suffix $\perp$ has been placed to designate the force due to the external flow along the $y$-axis, the transverse direction.

The proposed conjecture is, of, course, subject to restrictions on the geometry of the meridional body profile $y(x)$ of continuously turning tangent implying that $y^{\prime}(x)$ is continuous together with $y^{\prime \prime}(x) \neq 0$, thereby avoiding corners or sharp edges or other kind of nodes and straight line portions, $y=a x+b, x_{1} \leq x \leq x_{2}$. Also, it should be noted here that the method holds good for convex axially symmetric bodies which possesses fore-aft symmetry about the equatorial axis perpendicular to the axis of symmetry(polar axis). Apart from this argument, It is interesting to note here that the proposed conjecture is applicable also to those axi-symmetric bodies which fulfills the condition of continuously turning tangent but does not possesses fore-aft symmetry like egg shaped body (Datta \& Srivastava [1, 1999]). By using these formulae of drag, Srivastava et al. [30, (2012] have calculated the expressions of drag on the deformed sphere up to second order deformation parameter and later on oblate spheroid and flat circular disk as a special case. This conjecture is much simpler to evaluate the numerical values of drag than other existing numerical methods like Boundary Element Method (BEM), Finite Element Analysis (FEA) etc. as it can be applied to a large set of convex axi-symmetric bodies possessing fore-aft symmetry about maximal radius situated in the middle of the body for which analytical solution is not available or impossible to evaluate.

Since both axial and transverse flows have been considered in a free stream results of the force at an oblique angle of attack may be resolved into its components to get 
the required result. The present analysis can be extended to generate a drag formula for axi- symmetric bodies for more complex flows like paraboloidal flow for which free stream may be represented by surface average velocity (Chwang and $\mathrm{Wu}[2$, part 2, 1974]). The present analysis may be extended to generate a drag formula for axisymmetric bodies for more complex flows like paraboloidal flow and stagnation like paraboloidal flow for which free stream may be represented by surface average velocity (Chwang and $\mathrm{Wu}[2$, part 2, 1974]).

\section{Formulation of the Problem}

(Chwang and $\mathrm{Wu}[2$, part 2, 1974]) have constructed the exact solutions to a number of exterior and interior Stokes-flow problems by employing fundamental singularities (Stokeslets, rotlets, stresslets, potential doublets and higher order poles) for several specific body shapes translating and rotating in a flow other than primary flow include the uniform stream, shear flows, parabolic profiles and extensional flows(hyperbolic profiles), while the body shapes cover prolate spheroids, spheres and circular cylinders. Just after this paper, Chwang [3, part 3, 1975]) extended the analysis and considered the quadratic flows including unidirectional paraboloidal flows, with either an elliptic or a hyperbolic velocity distribution, and stagnation-like quadratic flows as typical representations. Each of these exact solutions (in closed form) regarding the types of singularities required the construction of a solution in each specific form; their distribution densities are not easily attainable always.

The immediate aim of this problem is to reduce this complexity and to evaluate the exact solution(closed form) by using the DS conjecture (Datta \& Srivastava 1, 1999]). In Section 2, we have briefly explained the formulas for evaluating drag on axially symmetric bodies placed under axial flow (flow is along the axis of symmetry) and transverse flow(along the direction perpendicular to axis of symmetry) and in Section 4, we explain the extension of this conjecture further for quadratic flows including parabolic flow (Figures 2 and 3 and stagnation like parabolic flow Figures 5 and ??).

\section{QuAdratic Flow PAST AXIALly SYMmetric BOdies}

4.1. Axial paraboloid flow(or unbounded longitudinal paraboloidal flow). We consider an axi-symmetric body placed under a flow (Figure 2 with a paraboloidal velocity profile

$$
\begin{aligned}
U_{\text {paraboloid }} & =K\left(y^{2}+z^{2}\right) \\
\text { or } & =K\left(\alpha y^{2}+z^{2}\right)
\end{aligned}
$$

along the axis of symmetry which is the $x$-axis, where $K$ is arbitrary constant and $\alpha$ is also arbitrary constant may be positive or negative indicating that paraboloidal flow is either elliptic or hyperbolic. When $\alpha$ vanishes, the paraboloidal flow degenerates into a two-dimensional parabolic flow. For arbitrary positive values of $\alpha$, it represents Hagen-Poiseuille flow through a pipe of elliptic cross-section. If $\alpha=1$, it becomes 
a paraboloidal flow of revolution which corresponds to Heigen-Poiseuille flow in a circular tube. Hyperbolic paraboloidal flow $(\alpha<0)$ may not exist physically, but it can certainly serve as a local component of a more complicated flow field.

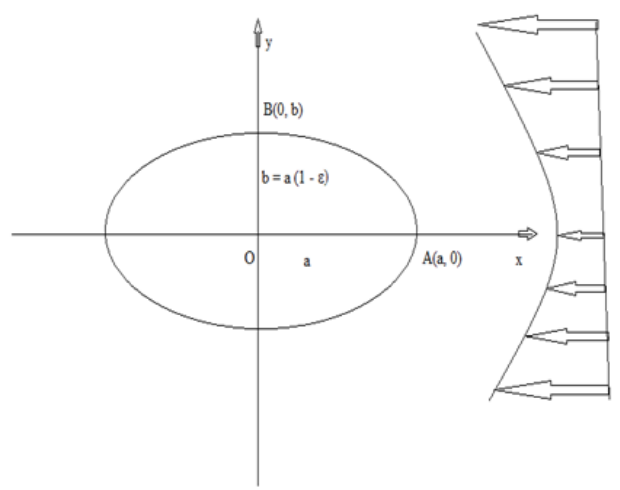

Figure 2. A longitudinal or axial paraboloidal flow past axisymmetric body

By DS-conjecture (Datta \& Srivastava [1, 1999]), the expression of drag on axially symmetric body placed in axial uniform stream $U$ (from equations $(2.2)$ and $(2.3)$ ) is

$$
\mathrm{F}_{x}=\frac{1}{2} \frac{\lambda b^{2}}{h_{x}}, \quad \text { where } \quad \lambda=6 \pi \mu U_{x}
$$

and

$$
h_{x}=\left(\frac{3}{8}\right) \int_{0}^{\pi} R \sin ^{3} \alpha d \alpha .
$$

where the suffix $x$ has been introduced to assert that the force is in the axial direction. Now equation (4.1) may be re-written as

$$
F_{x}=\frac{1}{2} \frac{(6 \pi \mu) b^{2}}{h_{x}} U_{x}=\frac{3 \pi \mu b^{2}}{h_{x}} U_{e},
$$

where $U_{e}$ is precisely the surface average of the primary flow velocity

$$
U=K r^{2}=K\left(y^{2}+z^{2}\right)
$$

over a surface of axi-symmetric body(conjectured by Chwang and $\mathrm{Wu}$ [2, part 2, 1975], $b$ is largest cross-section radius situated at the middle of the body and $h_{x}$ is given by equation 4.2 .

4.2. Transverse paraboloid flow. We consider an axi-symmetric body placed under a flow (Figure 3 ) with a paraboloidal velocity profile

$$
U_{\text {paraboloid }}=K\left(x^{2}+z^{2}\right)
$$




$$
\text { or }=\left(\beta x^{2}+z^{2}\right)
$$

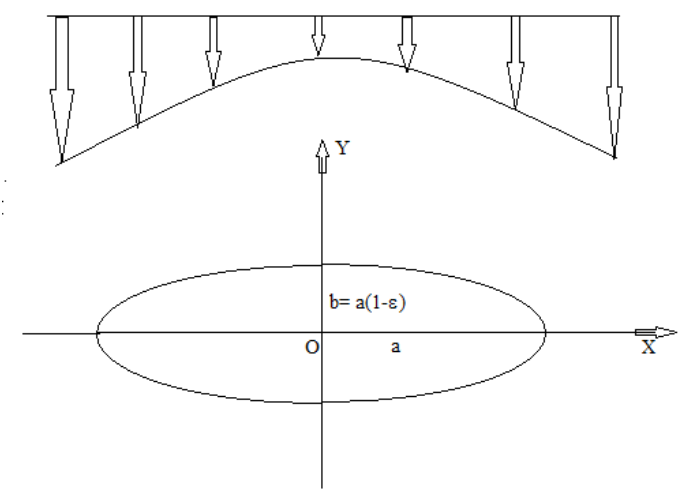

Figure 3. A transverse paraboloid flow past axisymmetric body

along axis of symmetry which is $y$-axis, where $K$ and $\beta$ are arbitrary constants.

The expression of Stokes drag on such type of axially symmetric bodies placed in transverse flow(uniform flow perpendicular to the axis of symmetry) is given by (Datta \& Srivastava [1, 1999])

$$
F_{y}=\frac{1}{2} \frac{\lambda b^{2}}{h_{y}} \quad \text { where } \quad \lambda=6 \pi \mu U_{y},
$$

and

$$
h_{y}=\left(\frac{3}{16}\right) \int_{0}^{\pi} R\left(2 \sin \alpha-\sin ^{3} \alpha\right) d \alpha .
$$

Now equation (4.7) may be re-written as

$$
F_{y}=\frac{1}{2} \frac{(6 \pi \mu) b^{2}}{h_{y}} U_{y}=\frac{3 \pi \mu b^{2}}{h_{y}} U_{e},
$$

where $U_{e}$ is precisely the surface average of the primary flow velocity

$$
U=K r^{2}=K\left(x^{2}+z^{2}\right)
$$

over a surface of axi-symmetric body (conjectured by Chwang and Wu 2, part 2, $1975]), b$ is largest cross-section radius situated at the middle of the body, and $h_{y}$ is given by equation 4.8.

Now, we apply the expressions 4.3 and 4.9 to evaluate drag on axially symmetric bodies like sphere, spheroid and egg-shaped body placed under longitudinal unbounded paraboloid and transverse unbounded paraboloid flows. 


\section{SPHERE}

5.1. Axial Flow. We shall consider a sphere having a radius a placed under axial paraboloidal flow velocity $U_{\text {paraboloid }}=K\left(y^{2}+z^{2}\right)$, by considering the surface average velocity $U_{e}=2 K a^{2} / 3$, over a spherical surface $R=a$, the value of $h_{x}=a / 2$ (from equation (4.2), the expression of drag on comes out to be (with the use of equation (4.3)

$$
F_{x}=\frac{3 \pi \mu b^{2}}{h_{x}} U_{e}=\frac{3 \pi \mu a^{2}}{\frac{a}{2}}\left(\frac{2}{3} K a^{2}\right)=4 \pi \mu K a^{3} .
$$

which matches with that obtained by Chwang and Wu ([2, part 2, 1975, p. 807, Eq. (69)]) treated with singularity method.

5.2. Transverse flow. Here we shall consider again a sphere having a radius $a$ placed under transverse paraboloidal flow velocity $U_{\text {paraboloid }}=K\left(x^{2}+z^{2}\right)$, by considering the surface average velocity $U_{e}=2 K a^{2} / 3$, over a spherical surface $R=a$, the value of $h_{y}=a / 2$ (from equation (4.8)), the expression of drag on sphere comes out to be (with the use of equation 4.3)

$$
F_{y}=\frac{3 \pi \mu b^{2}}{h_{y}} U_{e}=\frac{3 \pi \mu a^{2}}{\frac{a}{2}}\left(\frac{2}{3} K a^{2}\right)=4 \pi \mu K a^{3} .
$$

\section{Prolate SPheroid}

6.1. Axial Flow. We consider a prolate spheroid

$$
\frac{x^{2}}{a^{2}}+\frac{\rho^{2}}{b^{2}}=1, \quad \rho^{2}=y^{2}+z^{2}, \quad a \geq b, \quad c=\sqrt{a^{2}-b^{2}}=e a, \quad 0 \leq e \leq 1
$$

having semi-major axis $a$ and semi-minor axis $b, e$ as eccentricity, and $c$ as distance of focus from centre, placed under axial paraboloidal flow velocity $U_{\text {paraboloid }}=\left(\alpha y^{2}+\right.$ $z^{2}$ ) along $\mathrm{x}$-axis, and for $\alpha \neq 1$, the surface average velocity $U_{e}$, over a spheroidal surface 6.1 is

$$
U_{e}=\frac{1}{3}(1+\alpha)\left(1-e^{2}\right) a^{2},
$$

the value of $h_{x}$ (from equation 4.2 ) is

$$
h_{x}=\frac{3 b^{2}}{16 a e^{3}}\left[-2 e+\left(1+e^{2}\right) L\right], \quad L=\log \frac{1+e}{1-e} .
$$

For $\alpha \neq 1$, the expression of drag on prolate spheroid (6.1) in axial or longitudinal paraboloid flow may be obtained from 4.3 by utilizing the values of $U_{e}$ from $(6.2)$ and $h_{x}$ from $(6.3)$ as

$$
F_{x}=\frac{16 \pi \mu(1+\alpha) e^{3} a^{3}\left(1-e^{2}\right)}{3\left[-2 e+\left(1+e^{2}\right) L\right]},
$$

the expression of drag coefficient, for $\alpha \neq 1$, with respect to sphere drag, $4 \pi \mu a^{3}$, may be written as

$$
C_{F_{x}}=\frac{F_{x}}{4 \pi \mu a^{3}}=\frac{4(1+\alpha) e^{3}\left(1-e^{2}\right)}{3\left[-2 e+\left(1+e^{2}\right) L\right]}
$$


the corresponding variation of drag coefficient with respect to eccentricity for various values of $\alpha$ are given in Table 1 and depicted in Figure 6 .

For $\alpha=1$, the case of axi-symmetrical paraboloidal flow, this expression of drag (6.4) reduces to the closed form [by using surface average velocity $U_{e}=2 a^{2}\left(1-e^{2}\right) / 3=$ $\left.2 b^{2} / 3\right]$ as

$$
F_{x}=\frac{32 \pi \mu e^{3} a^{3}\left(1-e^{2}\right)}{3\left[-2 e+\left(1+e^{2}\right) L\right]},
$$

which immediately reduces to $4 \pi \mu a^{3}$, i.e., the drag on sphere having radius $a$ in longitudinal paraboloid axisymmetric flow, as $e \rightarrow 0(b=a)$. This limiting value of drag on sphere may be used to write the expression of drag coefficient $C_{F x}$ as

$$
C_{F_{x}}=\frac{F_{x}}{4 \pi \mu a^{3}}=\frac{8 e^{3}\left(1-e^{2}\right)}{3\left[-2 e+\left(1+e^{2}\right) L\right]} .
$$

Equation $6.5 \mathrm{~b}$ may be directly obtained by using $6.4 \mathrm{~b}$ and setting $\alpha$ to 1.

6.2. Transverse flow. We consider a prolate spheroid having semi-major axis $a$ and semi-minor axis $b, e$ as eccentricity, and $c$ as distance of focus from centre, placed under transverse paraboloidal flow velocity $U_{\text {paraboloid }}=\left(\beta x^{2}+z^{2}\right)$ along $y$-axis, and for $\beta \neq 1$, the surface average velocity $U_{e}$, over a spheroidal surface $(6.1)$ is

$$
U_{e}=\frac{1}{3}\left(1+\beta-e^{2}\right) a^{2}
$$

the value of $h_{y}$ (from equation (4.8) is

$$
h_{y}=\frac{3 b^{2}}{32 a e^{3}}\left[2 e+\left(3 e^{2}-1\right) L\right], \quad L=\log \frac{1+e}{1-e} .
$$

For $\beta \neq 1$, the expression of drag on prolate spheroid 6.1 may be obtained from (4.9) by utilizing the values of $U_{e}$ from (6.6) and $h_{y}$ from (6.7) as

$$
F_{y}=\frac{32 \pi \mu e^{3} a^{3}\left(1+\beta-e^{2}\right)}{3\left[2 e+\left(3 e^{2}-1\right) L\right]},
$$

the expression of drag coefficient, for $\beta \neq 1$, with respect to sphere drag, $4 \pi \mu a^{3}$, may be written as

$$
C_{F_{y}}=\frac{F_{y}}{4 \pi \mu a^{3}}=\frac{8 e^{3}\left(1+\beta-e^{2}\right)}{3\left[2 e+\left(3 e^{2}-1\right) L\right]},
$$

the corresponding variation of drag coefficient with respect to eccentricity for various values of $\beta$ are given in Table 2 and depicted in Figure 7.

For $\beta=1$, the case of axi-symmetrical paraboloidal flow, this expression of drag (6.8) reduces to the closed form [by using surface average velocity $U_{e}=a^{2}\left(2-e^{2}\right) / 3=$ $\left.\left(a^{2}+b^{2}\right) / 3\right]$ as

$$
F_{y}=\frac{32 \pi \mu e^{3} a^{3}\left(2-e^{2}\right)}{3\left[2 e+\left(3 e^{2}-1\right) L\right]}
$$


which immediately reduces to $4 \pi \mu a^{3}$, i.e. the drag on sphere having radius $a$ in transverse paraboloid axisymmetric flow, as $e \rightarrow 0,(b=a)$. This limiting value of drag on sphere may be used to write the expression of drag coefficient $C_{F y}$ as

$$
C_{F_{y}}=\frac{F_{y}}{4 \pi \mu a^{3}}=\frac{8 e^{3}\left(2-e^{2}\right)}{3\left[2 e+\left(3 e^{2}-1\right) L\right]} .
$$

This expression $6.9 \mathrm{~b}$ may be directly obtained by $6.8 \mathrm{~b}$, by taking $\beta=1$.

\section{OBLATE SPHEROID}

7.1. Axial Flow. We consider an oblate spheroid

$$
\frac{x^{2}}{b^{2}}+\frac{\rho^{2}}{a^{2}}=1, \quad \rho^{2}=y^{2}+z^{2}, \quad a \geq b, \quad c=\sqrt{a^{2}-b^{2}}=e a, \quad 0 \leq e \leq 1
$$

[on interchanging the roles of $a$ and $b$ in equation [6.1] ], $e$ as eccentricity, and $c$ as distance of focus from centre, placed under axial paraboloidal flow velocity $U_{\text {paraboloid }}=\left(\alpha y^{2}+z^{2}\right)$ along $x$-axis, and for $\alpha \neq 1$ the surface average velocity $U_{e}$, over a spheroidal surface 7.1 is

$$
U_{e}=\frac{1}{3}(1+\alpha) a^{2}
$$

the value of $h_{x}$ (from equation 4.2 ) is

$$
h_{x}=\frac{3 a}{8 e^{3}}\left[e \sqrt{1-e^{2}}-\left(1-2 e^{2}\right) \sin ^{-1} e\right], \quad L=\log \frac{1+e}{1-e} .
$$

For $\alpha \neq 1$ the expression of drag on oblate spheroid (7.1) may be obtained from equation (4.3) by utilizing the values of $U_{e}$ from $(7.2)$ and $h_{x}$ from 7.3 as

$$
F_{x}=\frac{8 \pi \mu e^{3} a^{3}(1+\alpha)}{3\left[e \sqrt{1-e^{2}}-\left(1-2 e^{2}\right) \sin ^{-1} e\right]},
$$

the expression of drag coefficient, for $\alpha \neq 1$, with respect to sphere drag, $4 \pi \mu a^{3}$, may be written as

$$
C_{F_{x}}=\frac{F_{x}}{4 \pi \mu a^{3}}=\frac{2 e^{3}(1+\alpha)}{3\left[e \sqrt{1-e^{2}}-\left(1-2 e^{2}\right) \sin ^{-1} e\right]}
$$

the corresponding variation of drag coefficient with respect to eccentricity for various values of $\alpha$ are given in Table 3 and depicted in Figure 8

For $\alpha=1$, the case of axi-symmetrical paraboloidal flow, this expression of drag (7.4) reduces to the closed form [by using surface average velocity $U_{e}=2 a^{2} / 3$ ] as

$$
F_{x}=\frac{16 \pi \mu e^{3} a^{3}}{3\left[e \sqrt{1-e^{2}}-\left(1-2 e^{2}\right) \sin ^{-1} e\right]},
$$

which immediately reduces to $4 \pi \mu a^{3}$, i.e. the drag on sphere having radius $a$ in longitudinal paraboloid axisymmetric flow, as $e \rightarrow 0(b=a)$. This limiting value of drag on sphere may be used to write the expression of drag coefficient $C_{F x}$ as

$$
C_{F_{x}}=\frac{F_{x}}{4 \pi \mu a^{3}}=\frac{4 e^{3}}{3\left[e \sqrt{1-e^{2}}-\left(1-2 e^{2}\right) \sin ^{-1} e\right]}
$$


Expression $(7.5 \mathrm{~b}$ may be directly obtained by $(7.4 \mathrm{~b})$, by taking $\alpha=1$.

7.2. Transverse flow. We consider a oblate spheroid having semi-major axis $b$ and semi-minor axis $a, e$ as eccentricity, and $c$ as distance of focus from centre, placed under axial paraboloidal flow velocity $U_{\text {paraboloid }}=\left(\beta x^{2}+z^{2}\right)$ along $y$-axis, and for $\beta \neq 1$, the surface average velocity $U_{e}$, over a spheroidal surface 6.1 is

$$
U_{e}=\frac{1}{3}\left(1+\beta-e^{2}\right) b^{2},
$$

the value of $h_{y}$ (from equation (4.8) ) is

$$
h_{y}=\frac{3 a}{16 e^{3}}\left[\left(1+2 e^{2}\right) \sin ^{-1} e-e \sqrt{1-e^{2}}\right], \quad L=\log \frac{1+e}{1-e} .
$$

For $\beta \neq 1$, the expression of drag on oblate spheroid (7.1) may be obtained from 4.9) by utilizing the values of $\mathrm{U}_{e}$ from $(7.6)$ and $\mathrm{h}_{x}$ from (7.7) as

$$
F_{y}=\frac{16 \pi \mu e^{3} a^{3}\left(1+\beta-e^{2}\right)}{3\left[\left(1+2 e^{2}\right) \sin ^{-1} e-e \sqrt{1-e^{2}}\right]},
$$

the expression of drag coefficient, for $\beta \neq 1$, with respect to sphere drag, $4 \pi \mu a^{3}$, may be written as

$$
C_{F_{y}}=\frac{F_{y}}{4 \pi \mu a^{3}}=\frac{4 e^{3}\left(1+\beta-e^{2}\right)}{3\left[\left(1+2 e^{2}\right) \sin ^{-1} e-e \sqrt{1-e^{2}}\right]}
$$

the corresponding variation of drag coefficient with respect to eccentricity for various values of $\beta$ are given in Table 4 and depicted in Figure 9.

For $\beta=1$, the case of axi-symmetrical paraboloidal flow, this expression of drag 7.8 reduces to the closed form [by using surface average velocity $U_{e}=b^{2}\left(2-e^{2}\right) / 3=$ $\left.\left(a^{2}+b^{2}\right) / 3\right]$ as

$$
F_{y}=\frac{16 \pi \mu e^{3} a b^{2}\left(2-e^{2}\right)}{3\left[\left(1+2 e^{2}\right) \sin ^{-1} e-e \sqrt{1-e^{2}}\right]},
$$

which immediately reduces to $4 \pi \mu a^{3}$, i.e. the drag on sphere having radius $a$ in transverse paraboloid axisymmetric flow, as $e \rightarrow 0(b=a)$. This limiting value of drag on sphere may be used to write the expression of drag coefficient $C_{F x}$ as

$$
C_{F_{y}}=\frac{F_{y}}{4 \pi \mu a^{3}}=\frac{4 e^{3}\left(2-e^{2}\right)}{3\left[\left(1+2 e^{2}\right) \sin ^{-1} e-e \sqrt{1-e^{2}}\right]} .
$$

This expression $7.9 \mathrm{~b}$ may be directly obtained by $7.8 \mathrm{~b}$, by taking $\beta=1$.

\section{EGG-SHAPED BODY}

8.1. Axial flow. We consider a body of revolution whose left half is semi-sphere with radius $b$

$$
x=b \cos t, \quad r=b \sin t, \quad \pi \leq t \leq \pi / 2,
$$

and right half is semi-spheroid (prolate) having semi-major axis length $a$ and semiminor axis length $b$

$$
x=a \cos t, \quad r=b \sin t, \quad \pi / 2 \leq t \leq 0
$$


placed under unbounded longitudinal or axial flow with parabolic velocity profile

$$
U_{\text {paraboloid }}=\alpha y^{2}+z^{2}
$$

along $x$-axis, and for $\alpha \neq 1$, the surface average velocity $U_{e}$ over egg-shaped body (8.1) is

$$
U_{e}=\frac{1}{6}(1+\alpha)\left[b^{2}+a^{2}\left(1-e^{2}\right)\right]
$$

the value of $h_{x}$ (from equation 4.2) is

$$
h_{x}=\frac{3}{8}\left\{\frac{2 b}{3}+\frac{b^{2}}{4 a e^{3}}\left[-2 e+\left(1+e^{2}\right) L\right]\right\}, \quad L=\log \frac{1+e}{1-e} .
$$

For $\alpha \neq 1$, the expression of drag on egg-shaped body (8.1) may be obtained from (4.3) by utilizing the values of $U_{e}$ from (8.2) and $h_{x}$ from (8.3) as

$$
F_{x}=\frac{8 \pi \mu a \sqrt{1-e^{2}}}{\frac{2}{3}+\frac{\sqrt{1-e^{2}}}{4 e^{3}}\left[-2 e+\left(1+e^{2}\right) L\right]}\left\{\frac{1}{6}(1+\alpha)\left[b^{2}+a^{2}\left(1-e^{2}\right)\right]\right\},
$$

the expression of drag coefficient, for $\alpha \neq 1$, with respect to sphere drag, $4 \pi \mu a^{3}$, may be written as [with the help of $b^{2}=a^{2}\left(1-e^{2}\right)$ ]

$$
C_{F_{x}}=\frac{F_{x}}{4 \pi \mu a^{3}}=\frac{4\left(1-e^{2}\right)^{3 / 2}}{2+3 \frac{\sqrt{1-e^{2}}}{4 e^{3}}\left[-2 e+\left(1+e^{2}\right) L\right]}
$$

the corresponding variation of drag coefficient with respect to eccentricity for various values of $\alpha$ are given in Table 5 and depicted in Figure 10.

For $\alpha=1$, equation 8.4a reduces to, by using the fact $b^{2}=a^{2}\left(1-e^{2}\right)$, the form

$$
F_{x}=\frac{16}{3} \frac{\pi \mu a^{3}\left(1-e^{2}\right)^{3 / 2}}{\left\{\frac{2}{3}+\frac{\sqrt{1-e^{2}}}{4 e^{3}}\left[-2 e+\left(1+e^{2}\right) L\right]\right\}},
$$

which immediately reduces to the expression of drag on sphere having radius $b$, i.e. $4 \pi \mu b^{3}$, as $e \rightarrow 0$. This limiting value of drag on sphere may be used to write the expression of drag coefficient $C_{F x}$ as

$$
C_{F_{x}}=\frac{F_{x}}{4 \pi \mu a^{3}}=\frac{4}{3} \frac{\left(1-e^{2}\right)^{3 / 2}}{\left\{\frac{2}{3}+\frac{\sqrt{1-e^{2}}}{4 e^{3}}\left[-2 e+\left(1+e^{2}\right) L\right]\right\}}
$$

This expression $8.5 \mathrm{~b}$ may be directly obtained by $8.4 \mathrm{~b}$, by taking $\alpha=1$.

As the drag on sphere and spheroid in paraboloid flow, $U_{\text {paraboloid }}=\alpha y^{2}+z^{2}$, has already been calculated independently in (5.1), (6.4) and (6.5), the expression of drag on egg-shaped body in paraboloid flow, for $\alpha \neq 1$, may be expressed in the combination of both as

$$
F_{x}=\pi \mu(1+) b^{3}+\frac{\pi \mu(1+\alpha)\left[8 e^{3} a^{3}\left(1-e^{2}\right)\right]}{3\left[-2 e+\left(1+e^{2}\right) L\right]},
$$


for $\alpha=1$, this expression 8.6 reduces to the form, with the aid of $b^{2}=a^{2}\left(1-e^{2}\right)$,

$$
F_{x}=2 \pi \mu \frac{b^{3}\left[-2 e+\left(1+e^{2}\right) L+8 e^{3} a^{3}\left(1-e^{2}\right)\right]}{-2 e+\left(1+e^{2}\right) L},
$$

which immediately reduces to the expression of drag on sphere having radius $b$ placed in axial axi-symmetric paraboloidal flow, i.e. $\pi \mu b^{3}$, as $e \rightarrow 0(a=b)$.

8.2. Transverse flow. We shall consider a body whose left half is semi-sphere with radius $b$

$$
x=b \cos t, \quad r=b \sin t, \quad \pi \leq t \leq \pi / 2,
$$

and right half is semi-spheroid(prolate) having semi-major axis length $a$ and semiminor axis length $b$

$$
x=a \cos t, \quad r=b \sin t, \quad \pi / 2 \leq t \leq 0,
$$

placed under unbounded transverse flow with parabolic velocity profile

$$
U_{\text {paraboloid }}=\beta x^{2}+z^{2}
$$

along $y$-axis, and for $\beta \neq 1$, the surface average velocity $U_{e}$ over egg-shaped body $(8.8)$ is

$$
U_{e}=\frac{\frac{1}{3}(1+\beta) b^{2}+\frac{1}{3}\left(1+\beta-e^{2}\right) b^{2}}{2}=\frac{b^{2}}{6}\left[2(1+\beta)-e^{2}\right]
$$

from where if $\beta=1$ it follows

$$
U_{e}=\frac{b^{2}}{6}\left(4-e^{2}\right) \rightarrow \frac{2}{3} b^{2}
$$

which is the average surface velocity on a sphere with radius $R=b$ as $e \rightarrow 0(b=a)$.

The value of $h_{y}$ from equation (4.8) is

$$
h_{y}=\frac{3}{16}\left[\frac{4}{3}+\frac{\sqrt{1-e^{2}}}{4 e^{3}}\left\{2 e+\left(3 e^{2}-1\right) L\right\}\right], \quad L=\log \frac{1+e}{1-e} .
$$

For $\beta \neq 1$, the expression of drag on egg-shaped body 8.8 may be obtained from (4.9) by utilizing the values of $U_{e}$ from 8.9 and $h_{y}$ from (8.10) as [with the aid of $\left.b^{2}=a^{2}\left(1-e^{2}\right)\right]$

$$
\begin{aligned}
F_{y}=\frac{16 \pi \mu a \sqrt{1-e^{2}}}{\left[\frac{4}{3}+\frac{\sqrt{1-e^{2}}}{4 e^{3}}\left\{2 e+\left(3 e^{2}-1\right) L\right\}\right]} & {\left[\frac{b^{2}}{6}\left\{2(1+\beta)-e^{2}\right\}\right]=} \\
& =\frac{8 \pi \mu a^{3}\left(1-e^{2}\right)^{3 / 2}\left[2(1+\beta) e^{2}\right]}{3\left[\frac{4}{3}+\frac{\sqrt{1-e^{2}}}{4 e^{3}}\left\{2 e+\left(3 e^{2}-1\right) L\right\}\right]}
\end{aligned}
$$

the expression of drag coefficient, for $\beta \neq 1$, with respect to sphere drag, $4 \pi \mu a^{3}$, may be written as

$$
C_{F_{y}}=\frac{F_{y}}{4 \pi \mu a^{3}}=\frac{2\left(1-e^{2}\right)^{3 / 2}\left[2(1+\beta)-e^{2}\right]}{3\left[\frac{4}{3}+\frac{\sqrt{1-e^{2}}}{4 e^{3}}\left\{2 e+\left(3 e^{2}-1\right) L\right\}\right]},
$$


the corresponding variation of drag coefficient with respect to eccentricity for various values of $\alpha$ are given in Table 6 and depicted in Figure 11 .

If $\beta=1$, equation $8.11 \mathrm{a}$ reduces to

$$
F_{y}=\frac{8 \pi \mu a^{3}\left(1-e^{2}\right)^{3 / 2}\left[4-e^{2}\right]}{3\left[\frac{4}{3}+\frac{\sqrt{1-e^{2}}}{4 e^{3}}\left\{2 e+\left(3 e^{2}-1\right) L\right\}\right]},
$$

which immediately reduces to the expression of drag on sphere having radius $b$, i.e. $4 \pi \mu b^{3}$, as $e \rightarrow 0(b=a)$. This limiting value of drag on sphere may be used to write the expression of drag coefficient $C_{F y}$ as

$$
C_{F_{y}}=\frac{F_{y}}{4 \pi \mu a^{3}}=\frac{2\left(1-e^{2}\right)^{3 / 2}\left[4-e^{2}\right]}{3\left[\frac{4}{3}+\frac{\sqrt{1-e^{2}}}{4 e^{3}}\left\{2 e+\left(3 e^{2}-1\right) L\right\}\right]} .
$$

Equation $8.12 \mathrm{~b}$ can also be obtained directly from $8.11 \mathrm{~b}$ by setting $\beta$ to 1 .

As the drag on sphere and spheroid in transverse paraboloid flow having velocity, $U_{\text {paraboloid }}=\beta x^{2}+z^{2}$, has already been calculated independently in $(5.2),(6.8)$ and 6.9 , the expression of drag on egg-shaped body in transverse paraboloid flow may be expressed in the combination of both as

$$
F_{y}=\pi \mu(1+\beta) b^{3}+\frac{16 \pi \mu e^{3} a^{3}\left(1+\beta-e^{2}\right)}{3\left[2 e+\left(3 e^{2}-1\right) L\right]} .
$$

If $\beta=1$ equation 8.13 takes the form

$$
F_{y}=\frac{2 \pi \mu\left[3 b^{3}\left\{2 e+\left(3 e^{2}-1\right) L\right\}+8 e^{3} a^{3}\left(2-e^{2}\right)\right]}{3\left[2 e+\left(3 e^{2}-1\right) L\right]},
$$

which immediately reduces to the drag expression of drag on sphere having radius $a$ placed in transverse axi-symmetric paraboloidal flow, i.e. $4 \pi \mu a^{3}$, as $e \rightarrow 0(b=a)$.

\section{Stagnation LiKe QUADRATIC FLOW PAST AXIALly SYMMETRIC BODIES}

9.1. Axial stagnation like paraboloid flow (or unbounded longitudinal stagnation paraboloidal flow). We consider an axi-symmetric body placed in a longitudinal stagnation-like quadratic flow (Figure 5 ) with velocity

$$
\begin{gathered}
\mathbf{u}=x^{2} \mathbf{e}_{x}-2 x y \mathbf{e}_{y} \\
u=|\mathbf{u}|=\left(x^{4}+4 x^{2} y^{2}\right)^{1 / 2}
\end{gathered}
$$

along axis of symmetry which is $x$-axis, which obviously satisfies the Stokes equations (2.1) if the pressure associated with it is $2 \mu x$. The stagnation plane is the centre-plane $x=0$. In the half-space $x<0$ the flow is towards the stagnation plane while in the half-space $x>0$ it is away from this plane. This type of quadratic flow is important since it can serve as a component in the general study of the motion of a spheroidal particle placed in a paraboloidal flow whose direction does not coincide with any one of the principal axes of the spheroid. 


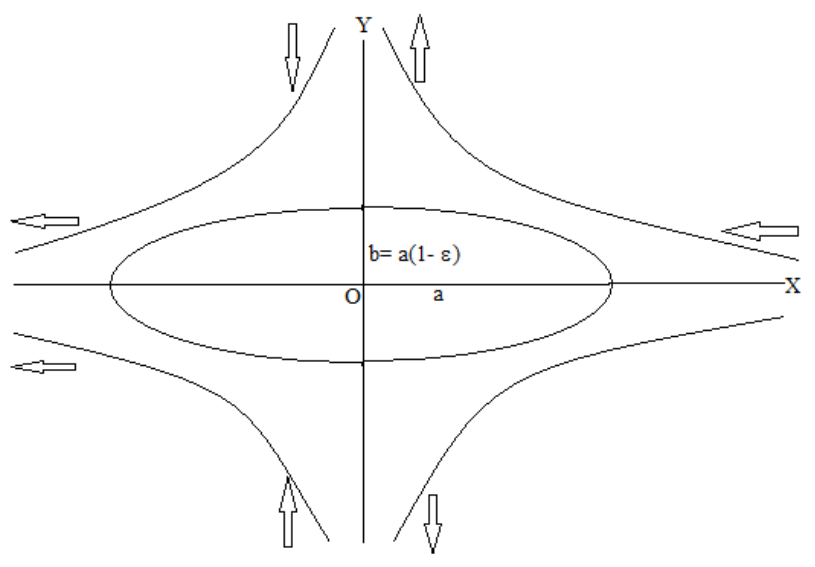

Figure 4. A longitudinal or axial stagnation like parabolic flow past axisymmetric body

9.2. Transverse stagnation like paraboloid flow. We consider an axi-symmetric body placed in a longitudinal stagnation-like quadratic flow (Figure 05 ) with velocity

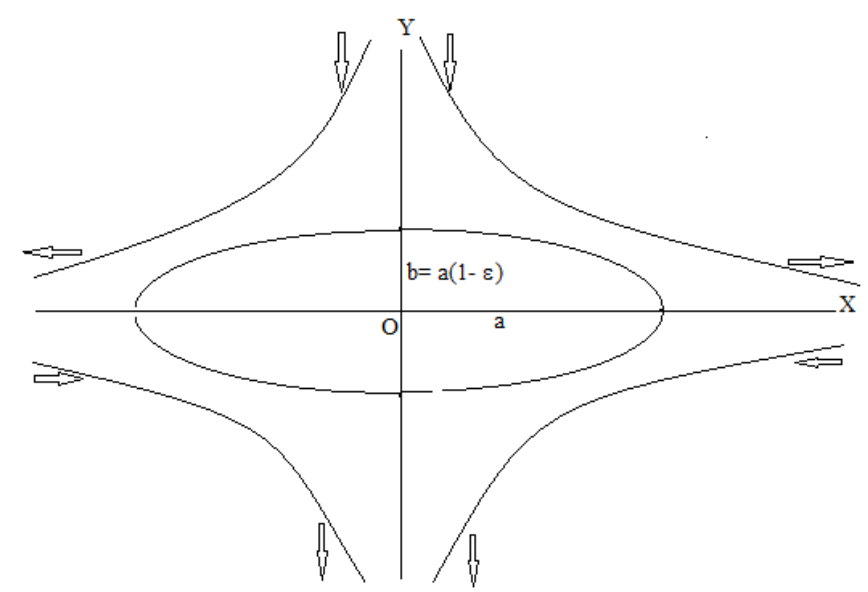

Figure 5. A longitudinal or axial stagnation like parabolic flow past axisymmetric body 


\section{SPHERE}

10.1. Axial Flow. We consider a sphere with radius $b$ and placed under axial stagnation like paraboloidal flow having velocity $U=|\mathbf{U}|=\left(x^{4}+4 x^{2} y^{2}\right)^{1 / 2}$, by considering the surface average velocity $U_{e}=b^{2} / 3$, over a spherical surface $R=b$, the value of $h_{x}=b / 2$ (from equation (4.2) ), the expression of drag on sphere comes out to be (with the use of equation 4.3 )

$$
F_{x}=\frac{3 \pi \mu b^{2}}{h_{x}} U_{e}=\frac{3 \pi \mu b^{2}}{\frac{a}{2}}\left(\frac{1}{3} b^{2}\right)=2 \pi \mu b^{3},
$$

which matches with that obtained by Chwang [3, part 3, 1975, p. 28, eq. 42] treated with singularity method.

10.2. Transverse flow. We consider a sphere having radius $b$ placed under transverse stagnation like paraboloidal flow velocity $U=|\mathbf{U}|=\left(y^{4}+4 x^{2} y^{2}\right)^{1 / 2}$, by considering the surface average velocity $U_{e}=(1 / 3) \mathrm{b}^{2}$, over a spherical surface $R=b$, the value of $h_{y}=b / 2$ (from equation 4.8), the expression of drag on sphere in transverse stagnation like paraboloidal flow comes out to be (with the use of equation (4.9))

$$
F_{y}=\frac{3 \pi \mu b^{2}}{h_{y}} U_{e}=\frac{3 \pi \mu b^{2}}{\frac{b}{2}}\left(\frac{1}{3} b^{2}\right)=2 \pi \mu b^{3},
$$

which matches with that obtained by Chwang [3, part 3, 1975, p. 28, eq. 42] treated with singularity method and is exactly the same as obtained in axial situation (10.1). The reason behind it is the fore and aft symmetry of the spherical body.

\section{Prolate SPheroid}

11.1. Axial Flow. details - which is subjected to an axial stagnation like a paraboloidal flow with a velocity $U=|\mathbf{U}|=\left(x^{4}+4 x^{2} y^{2}\right)^{1 / 2}$ along $x$-axis. The surface average velocity $U_{e}$, over a spheroidal surface is

$$
U_{e}=\frac{1}{3} a^{2}
$$

The value of $h_{x}$ can easily be obtained from equation 4.2 and coincides with equation 6.3 .

The expression for drag on a prolate spheroid (6.1) in axial stagnation like a paraboloid flow may be obtained from 4.3 by utilizing the values of $U_{e}$ from (11.1) and $h_{x}$ from 6.3 as

$$
F_{x}=\frac{16 \pi \mu e^{3} a^{3}}{3\left[-2 e+\left(1+e^{2}\right) L\right]},
$$

The above expression takes the form $2 \pi \mu a^{3}$, i.e., that of the drag on a sphere with radius $a$ and placed in axial stagnation type parabolic flow with velocity $U=|\mathbf{U}|=$ 
$\left(x^{4}+4 x^{2} y^{2}\right)^{1 / 2}$, as $e \rightarrow 0$. The expression of drag coefficient, with respect to sphere drag $2 \pi \mu a^{3}$, may be written as

$$
C_{F_{x}}=\frac{F_{x}}{2 \pi \mu a^{3}}=\frac{8 e^{3}}{3\left[-2 e+\left(1+e^{2}\right) L\right]}
$$

the corresponding variation of drag coefficient with respect to eccentricity are given in Table 7 and depicted in Figure 12

11.2. Transverse flow. Assume that the prolete spheroid is placed under transverse stagnation like a paraboloid flow having velocity $U=|\mathbf{U}|=\left(y^{4}+4 x^{2} y^{2}\right)^{1 / 2}$ along $y$-axis. The surface average velocity $U_{e}$ over a spheroidal surface is

$$
U_{e}=\frac{1}{3} b^{2}=\frac{1}{3} a^{2}\left(1-e^{2}\right)
$$

The value of $h_{y}$ (obtainable from equation (4.8) ) coincides with the right side of equation 6.7).

The expression of drag on prolate spheroid (6.1) in transverse stagnation like paraboloid flow can be obtained from $(4.9)$ by utilizing the values of $U_{e}$ from $(11.3)$ and $h_{y}$ from 6.7) as

$$
F_{y}=\frac{32 \pi \mu e^{3} a}{2 e+\left(3 e^{2}-1\right) L} \frac{a^{2}\left(1-e^{2}\right)}{3}=\frac{32 \pi \mu e^{3} a^{3}\left(1-e^{2}\right)}{3\left[2 e+\left(3 e^{2}-1\right) L\right]},
$$

which immediately reduces to $2 \pi \mu a^{3}$, the drag on sphere having a radius $a$ placed in transverse stagnation type parabolic flow with velocity $\left.U=|\mathbf{U}|=y^{4}+4 x^{2} y^{2}\right)^{1 / 2}$, as $e \rightarrow 0$. The expression of drag coefficient, with respect to sphere drag, $2 \pi \mu a^{3}$ may be written as

$$
C_{F_{y}}=\frac{F_{y}}{2 \pi \mu a^{3}}=\frac{16 e^{3}\left(1-e^{2}\right)}{3\left[2 e+\left(3 e^{2}-1\right) L\right]}
$$

the corresponding variation of drag coefficient with respect to eccentricity are given in Table 7 and is depicted in Figure 12 .

\section{Oblate SPheroid}

12.1. Axial Flow. We consider an oblate spheroid - see equation (7.1). If we interchange the roles of $a$ and $b$ in equation 6.1 - $e$ is eccentricity and $c$ is the distance of focus from centre - assuming axial paraboloidal flow velocity $U=|\mathbf{U}|=$ $\left(x^{4}+4 x^{2} y^{2}\right)^{1 / 2}$ along $x$-axis, the surface average velocity $U_{e}$, over the spheroidal surface (7.1) is

$$
U_{e}=\frac{1}{3} b^{2}
$$

The value of $h_{x}$ (obtainable from equation 4.2 ) coincides with the right side of equation 7.3 .

The expression of drag on oblate spheroid (7.1) in axial stagnation like paraboloid flow can be obtained from 4.3 by utilizing the values of $U_{e}$ from (11.1) and $h_{x}$ from 
(7.3) as

$$
F_{x}=\frac{8 \pi \mu a e^{3}}{e \sqrt{1-e^{2}}-\left(1-2 e^{2}\right) \sin ^{-1} e} \frac{b^{2}}{3}=\frac{8 \pi \mu a^{3} e^{3}\left(1-e^{2}\right)}{3\left[e \sqrt{1-e^{2}}-\left(1-2 e^{2}\right) \sin ^{-1} e\right]} .
$$

Equation of drag 12.2a reduces to the closed form, $2 \pi \mu b^{3}$, the drag on sphere having radius $b$ placed in axial stagnation type parabolic flow with velocity $U=|\mathbf{U}|=$ $\left(x^{4}+4 x^{2} y^{2}\right)^{1 / 2}$ as $e \rightarrow 0(b=a)$. The expression of drag coefficient, with respect to sphere drag, $2 \pi \mu a^{3}$, may be written as

$$
C_{F_{x}}=\frac{F_{x}}{2 \pi \mu a^{3}}=\frac{4 e^{3}\left(1-e^{2}\right)}{3\left[e \sqrt{1-e^{2}}-\left(1-2 e^{2}\right) \sin ^{-1} e\right]}
$$

the corresponding variation of drag coefficient with respect to eccentricity are given in Table 8 and depicted in Figure 13

12.2. Transverse flow. We consider an oblate spheroid (7.1), $e$ as eccentricity, and $a$ as distance of focus from centre, placed under transverse stagnation like paraboloid flow having velocity $U=U|\mathbf{U}|=\left(y^{4}+4 x^{2} y^{2}\right)^{1 / 2}$ along $y$-axis. The surface average velocity $U_{e}$, over a spheroidal surface 7.1 , is

$$
U_{e}=\frac{1}{3} a^{2}
$$

the value of $h_{y}$ (obtainable from equation 4.2 ) coincides with the right side of equation (7.7).

The expression of drag on oblate spheroid (7.1) in transverse stagnation like paraboloid flow may be obtained from 4.9 by utilizing the values of $U_{e}$ from 12.3 and $h_{y}$ from (7.7) as

$$
F_{y}=\frac{16 \pi \mu e^{3} a}{\left(1+2 e^{2}\right) \sin ^{-1} e-e \sqrt{1-e^{2}}} \frac{a^{2}}{3}=\frac{16 \pi \mu e^{3} a^{3}}{3\left[\left(1+2 e^{2}\right) \sin ^{-1} e-e \sqrt{1-e^{2}}\right]},
$$

which immediately reduces to $2 \pi \mu b^{3}$, the drag on sphere having radius $b$ placed in transverse stagnation type parabolic flow with velocity $U=|\mathbf{U}|=\left(y^{4}+4 x^{2} y^{2}\right)^{1 / 2}$, as $e \rightarrow 0(b=a)$. The expression of drag coefficient, with respect to sphere drag, $\pi \mu a^{3}$, may be written as

$$
C_{F_{y}}=\frac{F_{y}}{2 \pi \mu a^{3}}=\frac{8 e^{3}}{3\left[\left(1+2 e^{2}\right) \sin ^{-1} e-e \sqrt{1-e^{2}}\right]}
$$

the corresponding variation of drag coefficient with respect to eccentricity are given in Table 8 and depicted in Figure 13.

\section{EGG-SHAPED BODY}

13.1. Axial flow. We consider again the body described by equations (8.1) and placed under unbounded longitudinal or axial stagnation flow with parabolic velocity 
profile $U=|\mathbf{U}|=\left(x^{4}+4 x^{2} y^{2}\right)^{1 / 2}$ along $x$-axis. The surface average velocity $U_{e}$ over egg-shaped body 8.1 is

$$
U_{e}=\frac{1}{6}\left[b^{2}+a^{2}\left(1-e^{2}\right)\right]
$$

The value of $h_{x}$ (obtainable from equation (4.2) coincides with the right side of equation 8.2.

The expression of drag on egg-shaped body 8.1 may be obtained from 4.3 by utilizing the values of $U_{e}$ from (13.1) and $h_{x}$ from (8.2) as

$$
F_{x}=\frac{8 \pi \mu a \sqrt{1-e^{2}}}{\frac{2}{3}+\frac{\sqrt{1-e^{2}}}{4 e^{2}}\left\{-2 e+\left(1+e^{2}\right) L\right\}} \frac{1}{6}\left[b^{2}+a^{2}\left(1-e^{2}\right)\right] .
$$

Equation (??) reduces to, by using the fact $b^{2}=a^{2}\left(1-e^{2}\right)$, the form

$$
F_{x}=\frac{16}{3} \frac{\pi \mu a^{3}\left(1-e^{2}\right)^{3 / 2}}{\frac{2}{3}+\frac{\sqrt{1-e^{2}}}{4 e^{2}}\left[-2 e+\left(1+e^{2}\right) L\right]},
$$

The expression of drag coefficient, with respect to sphere drag, $2 \pi \mu a^{3}$, may be written as

$$
C_{F_{x}}=\frac{F_{x}}{2 \pi \mu a^{3}}=\frac{8\left(1-e^{2}\right)^{3 / 2}}{3\left\{\frac{2}{3}+\frac{\sqrt{1-e^{2}}}{4 e^{2}}\left[-2 e+\left(1+e^{2}\right) L\right]\right\}}
$$

the corresponding variation of drag coefficient with respect to eccentricity are given in Table 9 and depicted in Figure 14

Equation 13.3a immediately reduces to the expression of drag on sphere having radius $b$ placed in axial stagnation like paraboloid flow, i.e., $2 \pi \mu b^{3}$, as $e \rightarrow 0(b=a)$. As the drag on sphere and spheroid in axial stagnation type paraboloid flow, $U=$ $|\mathbf{U}|=\left(x^{4}+4 x^{2} y^{2}\right)^{1 / 2}$, has already been calculated independently in 10.1 and 11.2 . 11.4), the expression of drag on egg-shaped body in axial stagnation like paraboloid flow may be expressed in the combination of both as

$$
F_{x}=\pi \mu b^{3}+\frac{8 \pi \mu e^{3} a^{3}}{3\left[-2 e+\left(1+e^{2}\right) L\right]},
$$

which immediately reduces to the drag expression of drag on sphere having radius ' $b$ ' placed in axial stagnation like paraboloidal flow i.e. $2 \pi \mu b^{3}$, as $e \rightarrow 0,(b=a)$.

13.2. Transverse flow. We consider again the body described by equations 8.8 and placed under unbounded longitudinal or axial stagnation flow with under unbounded transverse stagnation like flow with parabolic velocity profile $U=|\mathbf{U}|=$ $\left(y^{4}+4 x^{2} y^{2}\right)^{1 / 2}$ along $y$-axis. The surface average velocity $U_{e}$ over egg-shaped body 8.8 is

$$
U_{e}=\frac{b^{2}}{6}\left(2-e^{2}\right)=\frac{1}{3} b^{2}
$$

i.e. the average surface velocity on sphere placed in stagnation like flow, as $e \rightarrow 0$.

The value of $h_{x}$ (obtainable from equation 4.2) coincides with the right side of equation 8.10. 
The expression of drag on egg-shaped body 8.8 may be obtained from 4.9 by utilizing the values of $U_{e}$ from 13.5 and $h_{y}$ from (8.10) as

$$
F_{y}=\frac{16 \pi \mu a \sqrt{1-e^{2}}}{\frac{4}{3}+\frac{\sqrt{1-e^{2}}}{4 e^{3}}\left[2 e+\left(3 e^{2}-1\right) L\right]} \frac{b^{2}}{6}\left(2-e^{2}\right)=\frac{8 \pi \mu a^{3}\left(1-e^{2}\right)^{3 / 2}\left(2-e^{2}\right)}{3\left\{\frac{4}{3}+\frac{\sqrt{1-e^{2}}}{4 e^{3}}\left[2 e+\left(3 e^{2}-1\right) L\right]\right\}},
$$

which immediately reduces to the expression of drag on sphere having radius $b$ placed in transverse stagnation like flow of paraboloidal velocity, $U=|\mathbf{U}|=\left(y^{4}+4 x^{2} y^{2}\right)^{1 / 2}$, i.e. $2 \pi \mu b^{3}$, as $e \rightarrow 0$.

The expression of drag coefficient, with respect to sphere drag, $2 \pi \mu a^{3}$, may be written as

$$
C_{F_{y}}=\frac{F_{y}}{2 \pi \mu a^{3}}=\frac{4\left(1-e^{2}\right)^{3 / 2}\left(2-e^{2}\right)}{3\left\{\frac{4}{3}+\frac{\sqrt{1-e^{2}}}{4 e^{3}}\left[2 e+\left(3 e^{2}-1\right) L\right]\right\}},
$$

the corresponding variation of drag coefficient with respect to eccentricity are given in Table 9 and depicted in Figure 14.

As the drag on sphere and spheroid in transverse stagnation like flow of paraboloidal velocity, $U=|\mathbf{U}|=\left(y^{4}+4 x^{2} y^{2}\right)^{1 / 2}$, has already been calculated independently in 10.2 and 11.4a), the expression of drag on egg-shaped body in transverse stagnation like paraboloid flow may be expressed in the combination of both as

$$
F_{y}=\pi \mu b^{3}+\frac{16 \pi \mu e^{3} a^{3}\left(1-e^{2}\right)}{3\left[2 e+\left(3 e^{2}-1\right) L\right]},
$$

which immediately reduces to the drag on sphere having radius $b$ placed in transverse stagnation like paraboloidal flow, $U=|\mathbf{U}|=\left(y^{4}+4 x^{2} y^{2}\right)^{1 / 2}$, i.e. $2 \pi \mu b^{3}$, as $e \rightarrow 0$, $(b=a)$.

\section{NUMERICAL DISCUSSION}

The numerical values of drag coefficient for axi-symmetric bodies like sphere, spheroid and egg-shaped body placed in axial and transverse quadratic (parabolic) flow have been calculated with respect to eccentricity $e$ and presented in Tables 1 6 respectively for various values of flow constants $\alpha$ and $\beta$ (varies from 1 to 5 ). In all these calculations, the flow constants $\alpha$ and $\beta$ are equal to one which represents the case of axi-symmetric quadratic Stokes flow. In Table 1, the values of $C_{F x}$ (drag coefficient for prolate spheroid placed in axial parabolic flow, Figure 2 decreases from corresponding drag values of sphere $(1.0,1.5,2.0,2.5,3.0$ for $\alpha=1,2,3,4,5)$ to 0 with respect to increasing values of eccentricity $e$ (from 0 to 1 ). In Table 1, it is interesting to note that the maximum difference between the two values of $C_{F x}$ for two successive values of flow constant $\alpha$ never exceeded by 0.5 which in every case decreases further to 0 . For $e=1$, the case of slender or needle shaped body, $C_{F x}$ comes out to be 0 which is justified. In comparison to axi-symmetric flow (for $\alpha=1$ ), the non-symmetric flow situations (for $\alpha=2,3,4,5, \ldots$ ) create maximum difference 
by values $0.5,1.0,1.5,2.0$ and so on. All the respective values of $C_{F x}$ have been calculated from equation $6.4 \mathrm{~b})$. All these variations are depicted in Figure 6 .

In Table 2 the values of $C_{F y}$ (drag coefficient for prolate spheroid placed in transverse parabolic flow, Figure 3) decreases from corresponding drag values of sphere $(1.0,1.5,2.0,2.5,3.0$ for $\beta=1,2,3,4,5)$ to 0 with respect to increasing values of eccentricity e(from 0 to 1 ). In Table 2 , it is interesting to note that the maximum difference between the two values of $C_{F y}$ for two successive values of flow constant $\beta$ never exceeded by 0.5 which in every case decreases further to 0 . For $e=1$, the case of slender or needle shaped body, $C_{F y}$ comes out to be 0 which is justified. In comparison to axi-symmetric flow (for $\beta=1$ ), the non-symmetric flow situations(for $\beta=2,3,4,5, \ldots)$ create maximum difference by values $0.5,1.0,1.5,2.0$ and so on. All the respective values of $C_{F y}$ have been calculated from equation $6.8 \mathrm{~b}$. On comparing the values of drag coefficient in Table 1 and Table 2, it may be observed that the transverse values are always greater then to axial values at every stage. This fact is also justified as the broader part of the prolate body faces the transverse flow. All these variations are depicted in Figure 7.

In Table 3 , the values of $C_{F x}$ (drag coefficient for oblate spheroid placed in axial parabolic flow, Figure 2 decreases from corresponding drag values of sphere(1.0, 1.5, $2.0,2.5,3.0$ for $\alpha=1,2,3,4,5)$ with respect to increasing values of eccentricity $e$ (from 0 to 1 ). In Table 3 it is interesting to note that the maximum difference between the two values of $C_{F x}$ for two successive values of flow constant $\alpha$ never exceeded by 0.5 which in every case decreases further. For $e=1$, the case of flat circular disk, $C_{F x}$ comes out to be non-zero which is justified again. In comparison to axi-symmetric flow (for $\alpha=1$ ), the non-symmetric flow situations (for $\alpha=2,3,4,5, \ldots$ ) create maximum difference by values $0.5,1.0,1.5,2.0$ and so on. All the respective values of $C_{F x}$ have been calculated from equation $7.4 \mathrm{~b}$ ). All these variations are depicted in Figure 8,

In Table 4 , the values of $C_{F x}$ (drag coefficient for oblate spheroid placed in transverse parabolic flow, Figure 2 decreases from corresponding drag values of sphere $(1.0,1.5,2.0,2.5,3.0$ for $\beta=1,2,3,4,5)$ with respect to increasing values of eccentricity e(from 0 to 1 ). In Table 4 , it is interesting to note that the maximum difference between the two values of $C_{F y}$ for two successive values of flow constant $\alpha$ never exceeded by 0.5 which in every case decreases further. For $\mathrm{e}=1$, the case of flat circular disk, $C_{F y}$ comes out to be non-zero which is justified again. In comparison to axisymmetric flow(for $\alpha=1$ ), the non-symmetric flow situations (for $\alpha=2,3,4,5, \ldots$ ) create maximum difference by values $0.5,1.0,1.5,2.0$ and so on. All the respective values of $C_{F x}$ have been calculated from equation $7.4 \mathrm{~b}$. On comparing the numerical values of drag coefficients for flat circular disk in axial and transverse flow cases, it may be observed that drag in axial flow is greater than drag in transverse flow. It is also justified the fact that broader part of oblate spheroid faces the axial paraboloid flow whereas the thinner portion faces the transverse paraboloid flow. All these variations are depicted in Figure 9. In Table 5 and 6, the numerical values of drag on egg-shaped body consisting of semi-spherical and semi-spheroidal part are presented. 


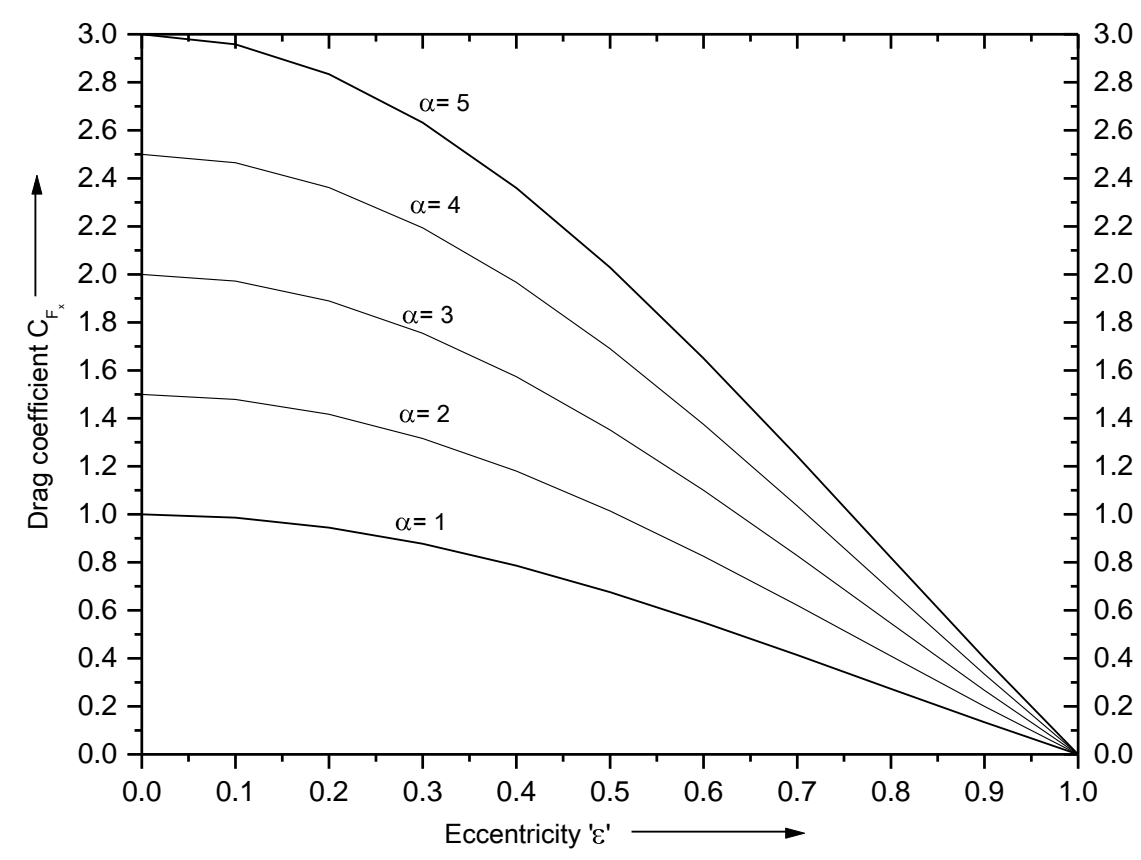

Figure 6. Variation of drag coefficient with respect to the eccentricity $e$ for various values of the constant $\alpha$

Table 1. Numerical values of drag coefficient(axial parabolic flow) for prolate spheroid with respect to eccentricity $e$ for various values of constant $\alpha$ (depicted in Figure 6) [Calculated from equation 6.4b)]

\begin{tabular}{|c|c|c|c|c|c|}
\hline & $\alpha=1$ & $\alpha=2$ & $\alpha=3$ & $\alpha=4$ & $\alpha=5$ \\
\hline $\mathrm{e}$ & $\mathrm{C}_{F x}$ & $\mathrm{C}_{F x}$ & $\mathrm{C}_{F x}$ & $\mathrm{C}_{F x}$ & $\mathrm{C}_{F x}$ \\
\hline \hline 0.0 & 1.000000 & 1.50000 & 2.0000 & 2.50000 & 3.00000 \\
\hline 0.1 & 0.986041 & 1.47906 & 1.9721 & 2.46513 & 2.95812 \\
\hline 0.2 & 0.944659 & 1.41699 & 1.8893 & 2.36165 & 2.83398 \\
\hline 0.3 & 0.877349 & 1.31602 & 1.7547 & 2.19337 & 2.63205 \\
\hline 0.4 & 0.786635 & 1.17995 & 1.5732 & 1.96659 & 2.35993 \\
\hline 0.5 & 0.676056 & 1.01408 & 1.3521 & 1.69014 & 2.02817 \\
\hline 0.6 & 0.550093 & 0.82514 & 1.1002 & 1.37523 & 1.65028 \\
\hline 0.7 & 0.413956 & 0.62093 & 0.8279 & 1.03489 & 1.24187 \\
\hline 0.8 & 0.273257 & 0.40989 & 0.5465 & 0.68314 & 0.81977 \\
\hline 0.9 & 0.133576 & 0.20036 & 0.2672 & 0.33394 & 0.40073 \\
\hline 1.0 & 0 & 0 & 0 & 0 & 0 \\
\hline
\end{tabular}




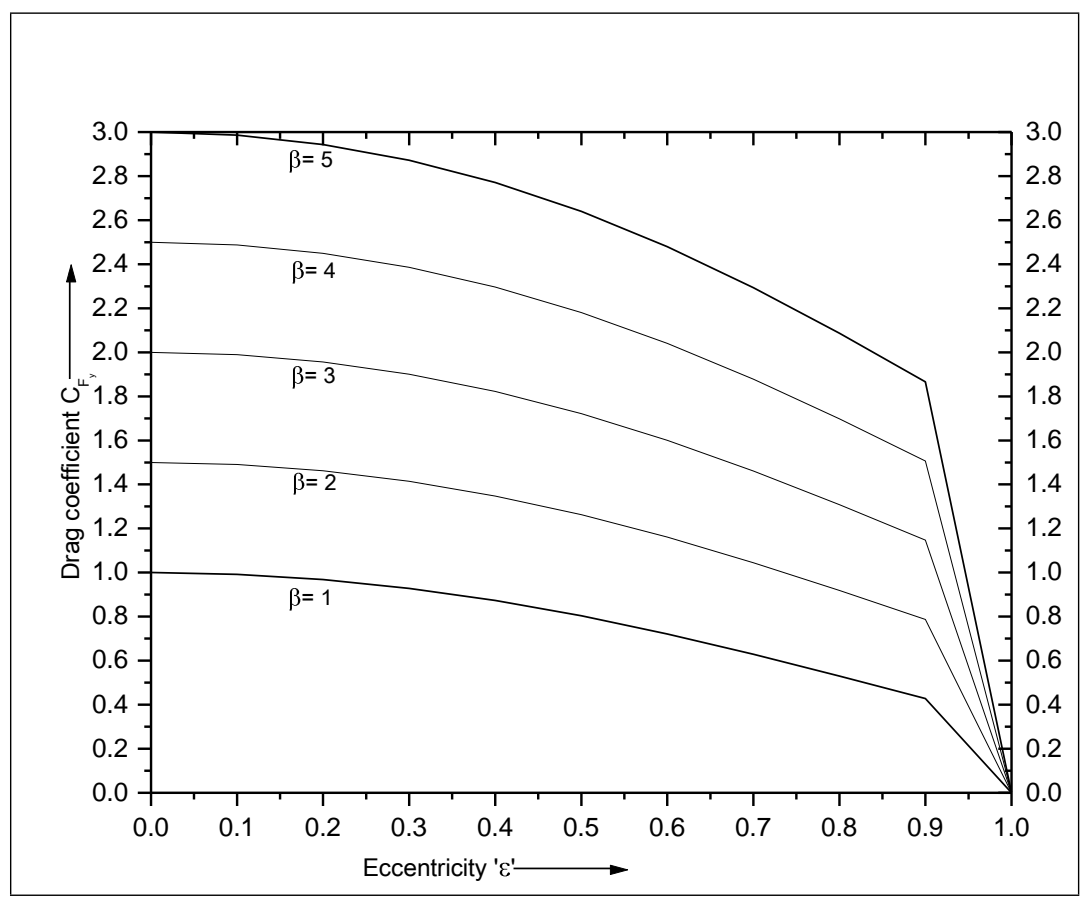

Figure 7. Variation of drag coefficient with respect to the eccentricity $e$ for various values of the constant $\beta$

Table 2. Numerical values of drag coefficient (transverse parabolic flow) for prolate spheroid with respect to eccentricity $e$ for various values of constant $\beta$ (depicted in Figure 7) [Calculated from equation $6.8 \mathrm{~b}]$

\begin{tabular}{|c|c|c|c|c|c|}
\hline & $\beta=1$ & $\beta=2$ & $\beta=3$ & $\beta=4$ & $\beta=5$ \\
\hline $\mathrm{e}$ & $\mathrm{C}_{F y}$ & $\mathrm{C}_{F y}$ & $\mathrm{C}_{F y}$ & $\mathrm{C}_{F y}$ & $\mathrm{C}_{F y}$ \\
\hline \hline 0.0 & 1.00000 & 1.50000 & 2.00000 & 2.50000 & 3.00000 \\
\hline 0.1 & 0.992002 & 1.49049 & 1.98899 & 2.48748 & 2.98597 \\
\hline 0.2 & 0.968035 & 1.46193 & 1.95583 & 2.44972 & 2.94362 \\
\hline 0.3 & $0 . .928246$ & 1.41424 & 1.90023 & 2.38622 & 2.87222 \\
\hline 0.4 & 0.873064 & 1.34756 & 1.82205 & 2.29654 & 2.77103 \\
\hline 0.5 & 0.803443 & 1.26255 & 1.72166 & 2.18077 & 2.63989 \\
\hline 0.6 & 0.721095 & 1.16079 & 1.60048 & 2.04017 & 2.47986 \\
\hline 0.7 & 0.628631 & 1.04494 & 1.46125 & 1.87757 & 2.29388 \\
\hline 0.8 & 0.529529 & 0.918889 & 1.30825 & 1.69761 & 2.08697 \\
\hline 0.9 & 0.427866 & 0.787417 & 1.14697 & 1.50652 & 1.86607 \\
\hline 1.0 & 0 & 0 & 0 & 0 & 0 \\
\hline
\end{tabular}




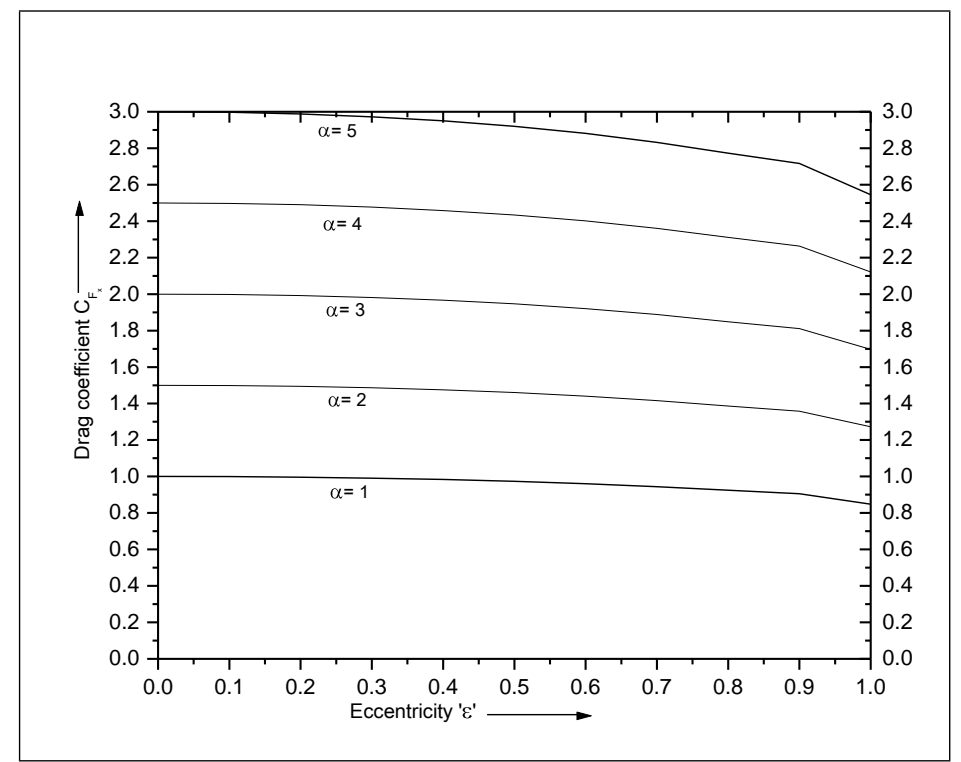

Figure 8. Variation of drag coefficient with respect to the eccentricity $e$ for various flow constant $\alpha$

Table 3. Variation of drag coefficient (axial parabolic flow) for oblate spheroid with respect to eccentricity $e$ for various values of constant $\alpha$ (depicted in Figure 8) [Calculated from equation (7.4b) )]

\begin{tabular}{|c|c|c|c|c|c|}
\hline & $\alpha=1$ & $\alpha=2$ & $\alpha=3$ & $\alpha=4$ & $\alpha=5$ \\
\hline $\mathrm{e}$ & $\mathrm{C}_{F x}$ & $\mathrm{C}_{F x}$ & $\mathrm{C}_{F x}$ & $\mathrm{C}_{F x}$ & $\mathrm{C}_{F x}$ \\
\hline \hline 0.0 & 1.00000 & 1.50000 & 2.0000 & 2.50000 & 3.00000 \\
\hline 0.1 & 0.998998 & 1.49856 & 1.9985 & 2.49749 & 2.99699 \\
\hline 0.2 & 0.995964 & 1.49395 & 1.9919 & 2.48991 & 2.98789 \\
\hline 0.3 & 0.990812 & 1.48622 & 1.9816 & 2.47703 & 2.97244 \\
\hline 0.4 & 0.983382 & 1.47507 & 1.9668 & 2.45846 & 2.95015 \\
\hline 0.5 & 0.973406 & 1.46011 & 1.9468 & 2.43351 & 2.92022 \\
\hline 0.6 & 0.960476 & 1.44071 & 1.9210 & 2.40119 & 2.88143 \\
\hline 0.7 & 0.944111 & 1.41617 & 1.8882 & 2.36028 & 2.83233 \\
\hline 0.8 & 0.924349 & 1.38652 & 1.8487 & 2.31087 & 2.77305 \\
\hline 0.9 & 0.905344 & 1.35802 & 1.8107 & 2.26336 & 2.71603 \\
\hline 1.0 & 0.848484 & 1.27272 & 1.6968 & 2.12125 & 2.54526 \\
\hline
\end{tabular}




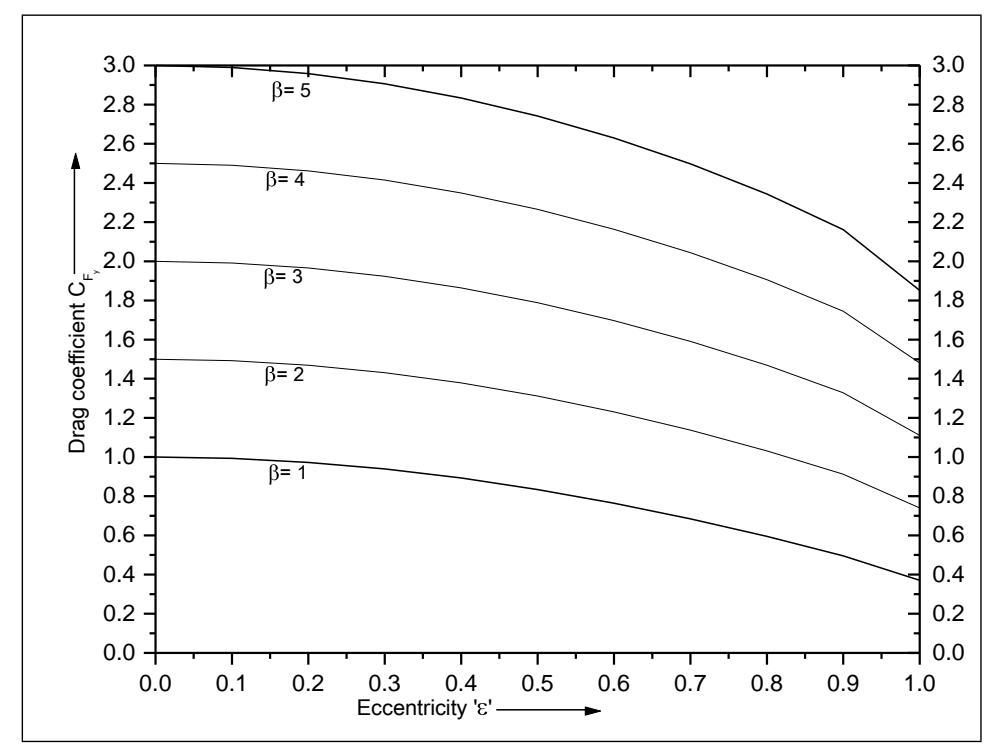

Figure 9. Variation of drag coefficient $C_{F_{x}}$ with respect to the eccentricity $e$ for various flow constant $\beta$

Table 4. Numerical values of drag coefficient(transverse parabolic flow) for oblate spheroid with respect to eccentricity $e$ for various values of constant $\beta$ (depicted in Figure 9) [Calculated from equation 7.8b]

\begin{tabular}{|c|c|c|c|c|c|}
\hline & $\beta=1$ & $\beta=2$ & $\beta=3$ & $\beta=4$ & $\beta=5$ \\
\hline $\mathrm{e}$ & $\mathrm{C}_{F x}$ & $\mathrm{C}_{F x}$ & $\mathrm{C}_{F x}$ & $\mathrm{C}_{F x}$ & $\mathrm{C}_{F x}$ \\
\hline \hline 0.0 & 1.000000 & 1.50000 & 2.00000 & 2.50000 & 3.00000 \\
\hline 0.1 & 0.993195 & 1.49229 & 1.99138 & 2.49047 & 2.98957 \\
\hline 0.2 & 0.972874 & 1.46924 & 1.96564 & 2.46197 & 2.95833 \\
\hline 0.3 & 0.939316 & 1.43114 & 1.92289 & 2.41468 & 2.90647 \\
\hline 0.4 & 0.892978 & 1.37829 & 1.86361 & 2.34892 & 2.83423 \\
\hline 0.5 & 0.834493 & 1.31135 & 1.78826 & 2.26505 & 2.74196 \\
\hline 0.6 & 0.764651 & 1.23095 & 1.69715 & 2.16344 & 2.62965 \\
\hline 0.7 & 0.684369 & 1.13759 & 1.59082 & 2.04404 & 2.49727 \\
\hline 0.8 & 0.594571 & 1.03176 & 1.46894 & 1.90612 & 2.34331 \\
\hline 0.9 & 0.495601 & 0.91207 & 1.32854 & 1.74501 & 2.16149 \\
\hline 1.0 & 0.370187 & 0.74037 & 1.11056 & 1.48074 & 1.85093 \\
\hline
\end{tabular}




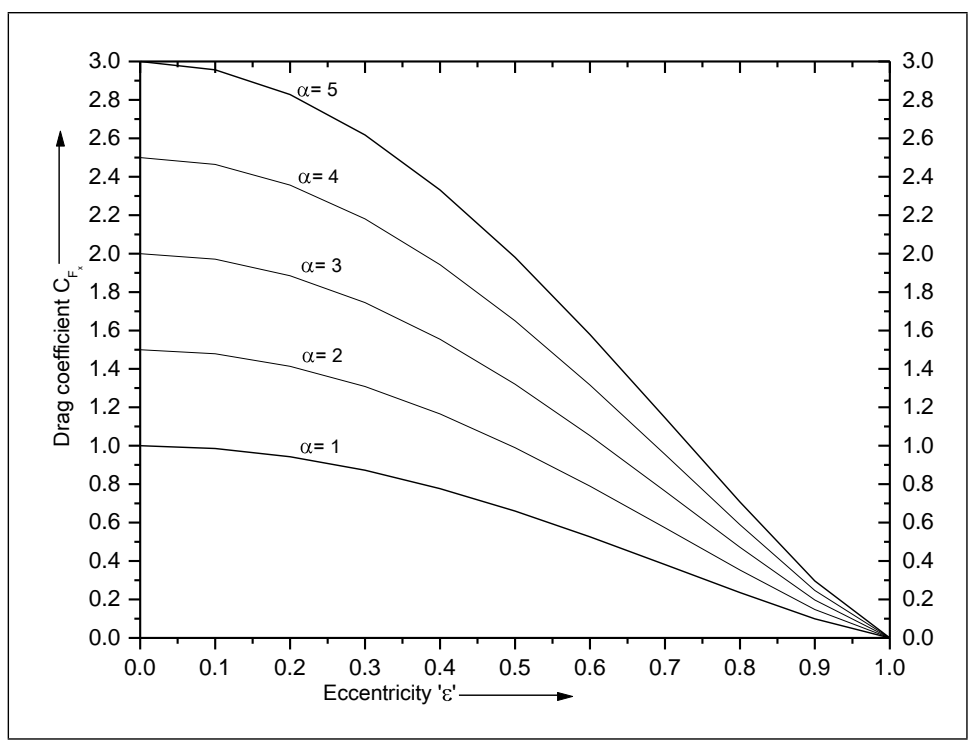

Figure 10. Variation of drag coefficient with respect to the eccentricity $e$ for various values of the constant $\alpha$

Table 5. Numerical values of drag coefficient (axial parabolic flow) for egg-shaped body with respect to eccentricity $e$ for various values of constant $\alpha$ (depicted in Figure 10] [Calculated from equation $8.4 \mathrm{~b}$ ]

\begin{tabular}{|c|c|c|c|c|c|}
\hline & $\alpha=1$ & $\alpha=2$ & $\alpha=3$ & $\alpha=4$ & $\alpha=5$ \\
\hline $\mathrm{e}$ & $\mathrm{C}_{F x}$ & $\mathrm{C}_{F x}$ & $\mathrm{C}_{F x}$ & $\mathrm{C}_{F x}$ & $\mathrm{C}_{F x}$ \\
\hline \hline 0.0 & 1.000000 & 1.50000 & 2.00000 & 2.50000 & 3.00000 \\
\hline 0.1 & 0.985534 & 1.47834 & 1.97107 & 2.46383 & 2.9568 \\
\hline 0.2 & 0.942545 & 1.41382 & 1.88509 & 2.35636 & 2.82763 \\
\hline 0.3 & 0.872297 & 1.30845 & 1.74459 & 2.18074 & 2.61689 \\
\hline 0.4 & 0.777018 & 1.16533 & 1.55404 & 1.94255 & 2.33105 \\
\hline 0.5 & 0.660082 & 0.99012 & 1.32016 & 1.65024 & 1.98025 \\
\hline 0.6 & 0.526246 & 0.78936 & 1.05249 & 1.31561 & 1.57874 \\
\hline 0.7 & 0.381933 & 0.57295 & 0.76386 & 0.95483 & 1.14586 \\
\hline 0.8 & 0.235596 & 0.35339 & 0.47119 & 0.58899 & 0.70679 \\
\hline 0.9 & 0.098860 & 0.14829 & 0.19772 & 0.24715 & 0.29658 \\
\hline 1.0 & 0 & 0 & 0 & 0 & 0 \\
\hline
\end{tabular}




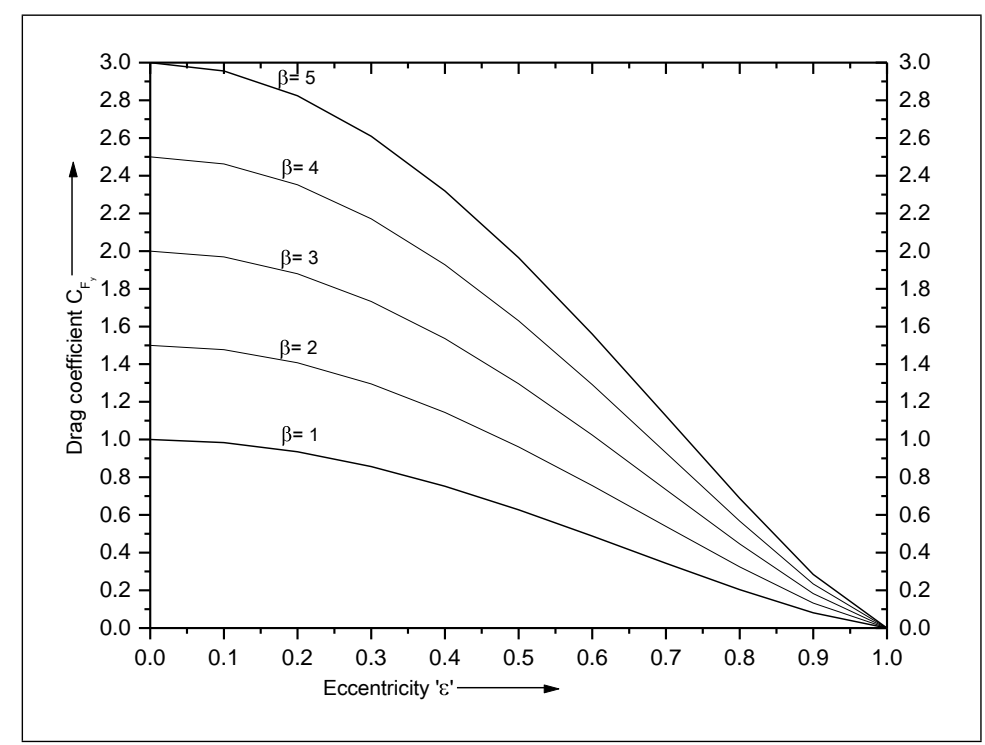

Figure 11. Variation of drag coefficient with respect to the eccentricity $e$ for various values of the constant $\beta$

Table 6. Numerical values of drag coefficient (transverse parabolic flow) for egg-shaped body with respect to eccentricity $e$ for various values of constant $\beta$ (depicted in Figure 11) [Calculated from equation $8.11 \mathrm{~b}$ ]

\begin{tabular}{|c|c|c|c|c|c|}
\hline & $\beta=1$ & $\beta=2$ & $\beta=3$ & $\beta=4$ & $\beta=5$ \\
\hline $\mathrm{e}$ & $\mathrm{C}_{F x}$ & $\mathrm{C}_{F x}$ & $\mathrm{C}_{F x}$ & $\mathrm{C}_{F x}$ & $\mathrm{C}_{F x}$ \\
\hline \hline 0.0 & 1.000000 & 1.50000 & 2.00000 & 2.50000 & 3.00000 \\
\hline 0.1 & 0.983564 & 1.47658 & 1.96959 & 2.46261 & 2.95562 \\
\hline 0.2 & 0.935017 & 1.40725 & 1.87948 & 2.35171 & 2.82394 \\
\hline 0.3 & 0.856634 & 1.29481 & 1.73299 & 2.17116 & 2.60934 \\
\hline 0.4 & 0.752191 & 1.14396 & 1.53572 & 1.92749 & 2.31926 \\
\hline 0.5 & 0.626971 & 0.96136 & 1.29574 & 1.63013 & 1.96451 \\
\hline 0.6 & 0.487834 & 0.75587 & 1.02391 & 1.29195 & 1.55998 \\
\hline 0.7 & 0.343286 & 0.53888 & 0.73448 & 0.93008 & 1.12568 \\
\hline 0.8 & 0.203567 & 0.32474 & 0.44591 & 0.56708 & 0.68825 \\
\hline 0.9 & 0.081137 & 0.13201 & 0.18288 & 0.23375 & 0.28462 \\
\hline 1.0 & 0 & 0 & 0 & 0 & 0 \\
\hline
\end{tabular}




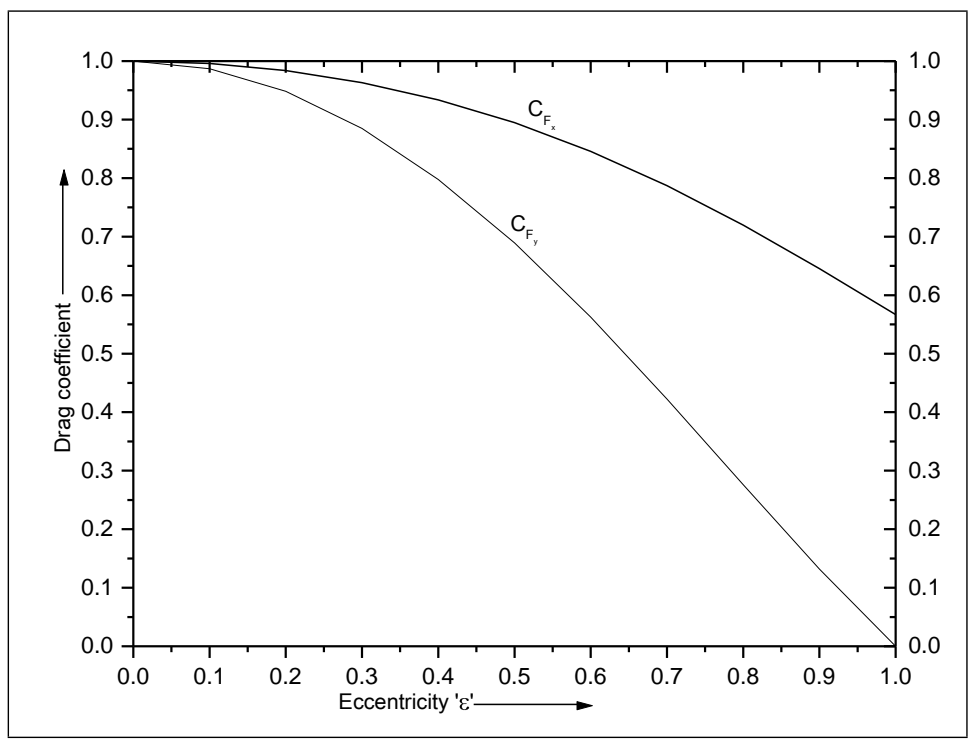

Figure 12. Variation of drag coefficient with respect to the eccentricity $e$ for prolate spheroid body placed in axial and transverse stagnation like quadratic flow

Table 7. Numerical values of drag coefficient (axial and transverse stagnation like quadratic flow) for prolate spheroid with respect to eccentricity $e$ (depicted in Figure 12) [Calculated from equations (11.2b) and (??)]

\begin{tabular}{|c|c|c|}
\hline & \multicolumn{2}{|c|}{$\begin{array}{c}\text { Txial } \\
\text { stagnation }\end{array}$} \\
\hline $\mathrm{e}$ & $\mathrm{C}_{F x}$ & $\mathrm{C}_{F y}$ \\
\hline \hline 0 & 1.000000 & 1.000000 \\
\hline 0.1 & 0.995990 & 0.987022 \\
\hline 0.2 & 0.983847 & 0.948350 \\
\hline 0.3 & 0.963242 & 0.884779 \\
\hline 0.4 & 0.933688 & 0.797685 \\
\hline 0.5 & 0.894658 & 0.689195 \\
\hline 0.6 & 0.845791 & 0.562531 \\
\hline 0.7 & 0.787188 & 0.422511 \\
\hline 0.8 & 0.719729 & 0.276000 \\
\hline 0.9 & 0.645306 & 0.131863 \\
\hline 1 & 0.566800 & 0.000000 \\
\hline
\end{tabular}




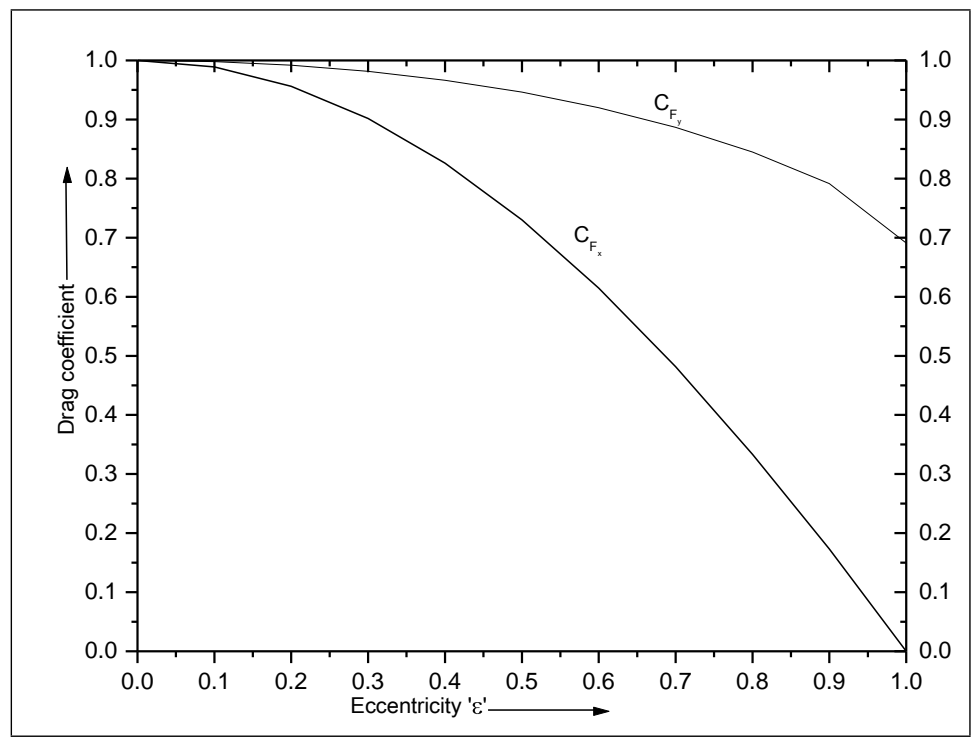

Figure 13. Variation of drag coefficient with respect to the eccentricity $e$ for oblate spheroid body placed in axial and transverse stagnation like quadratic flow

Table 8. Numerical values of drag coefficient (axial and transverse stagnation like quadratic flow) for oblate spheroid with respect to eccentricity $e$ (depicted in Figure 13) [Calculated from equations 12.2b) and (??)]

\begin{tabular}{|c|c|c|}
\hline & \multicolumn{2}{|c|}{$\begin{array}{c}\text { Axial } \\
\text { stagnation }\end{array}$} \\
\hline $\mathrm{e}$ & $\mathrm{C}_{F x}$ & $\mathrm{C}_{F y}$ \\
\hline \hline 0 & 1.000000 & 1.000000 \\
\hline 0.1 & 0.989008 & 0.997994 \\
\hline 0.2 & 0.956124 & 0.991909 \\
\hline 0.3 & 0.901627 & 0.981531 \\
\hline 0.4 & 0.82599 & 0.966489 \\
\hline 0.5 & 0.729928 & 0.946228 \\
\hline 0.6 & 0.614529 & 0.919985 \\
\hline 0.7 & 0.481472 & 0.886711 \\
\hline 0.8 & 0.333281 & 0.844916 \\
\hline 0.9 & 0.173193 & 0.791535 \\
\hline 1 & 0.000000 & 0.6910 \\
\hline
\end{tabular}




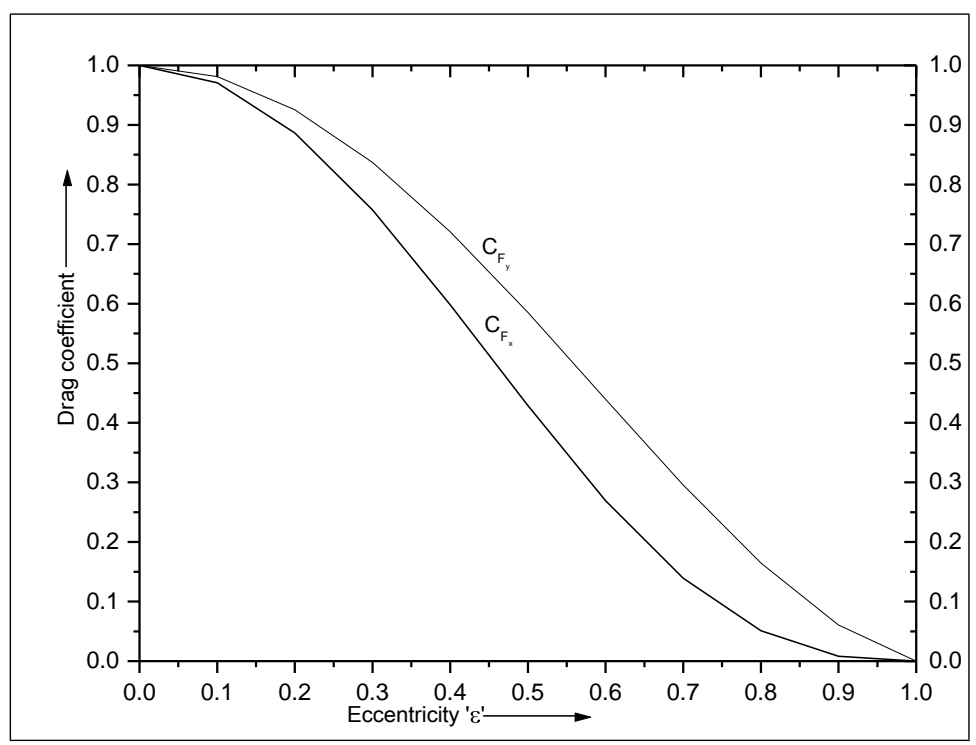

Figure 14. Variation of drag coefficient with respect to the eccentricity $e$ for egg-shaped body placed in axial and transverse stagnation like quadratic flow

Table 9. Numerical values of drag coefficient (axial and transverse stagnation like quadratic flow) for egg-shapped body with respect to eccentricity $e$ (depicted in Figure 14) [Calculated from equations 13.3b and 13.6b]

\begin{tabular}{|c|c|c|}
\hline & \multicolumn{2}{|c|}{$\begin{array}{c}\text { Axial } \\
\text { stagnation }\end{array}$} \\
\hline $\mathrm{e}$ & $\mathrm{C}_{F x}$ & $\mathrm{C}_{F y}$ \\
\hline \hline 0 & 1.0000000 & 1.000000 \\
\hline 0.1 & 0.9707880 & 0.981099 \\
\hline 0.2 & 0.8865610 & 0.925572 \\
\hline 0.3 & 0.7572270 & 0.836916 \\
\hline 0.4 & 0.5982050 & 0.72085 \\
\hline 0.5 & 0.4287360 & 0.585173 \\
\hline 0.6 & 0.2694380 & 0.439583 \\
\hline 0.7 & 0.1391050 & 0.295358 \\
\hline 0.8 & 0.05088870 & 0.164792 \\
\hline 0.9 & 0.00818752 & 0.0605348 \\
\hline 1 & 0.0000000 & 0.0000000 \\
\hline
\end{tabular}


For axisymmetric case $(\alpha=1)$, drag value decreases from 1 to 0 with respect to increasing eccentricity from 0 to 1 . For non-symmetric cases $(\alpha=2$ to 5$)$, for specific body, drag value increases on moving from left to right in table. In both the flows (axial and transverse), for $e=1$, the limiting case of slender body, drag comes out to be 0 , which is again justified. It clearly indicates that for uniform and parabolic flows the drag on slender needle shaped body, drag comes out to be same. The variation of these drag values with respect to eccentricity $e$ for various flow parameters $\alpha$ and $\beta$ are depicted in Figure 10 and 11, respectively.

In Table 7, the numerical values of drag on prolate spheroid in axial and transverse stagnation like quadratic flow have been calculated with respect to eccentricity $e$. It is found that drag values decreases from 1 to 0.56680 in axial flow situation and decreases from 1 to 0 in transverse situation as eccentricity $e$ increases from 0 to 1 . It is interesting to observe that in the limiting case as $e=1$, the case of slender or needle case, drag comes out to be non-zero $(=0.56680)$ while its value is zero in transverse flow situation. The variation of these drag values with respect to eccentricity $e$ are depicted in Figure 12

In Table 8, the numerical values of drag on oblate spheroid in axial and transverse stagnation like quadratic flow have been calculated with respect to eccentricity $e$. It is found that drag values decreases from 1 to 0 in axial flow situation and decreases from 1 to 0.6910 in transverse situation as eccentricity $e$ increases from 0 to 1 . It is interesting to observe that in the limiting case as $e=1$, the case of flat circular disk, drag comes out to be 0 while its value is non-zero $(=0.6910)$ in transverse flow situation. The variation of these drag values with respect to eccentricity $e$ are depicted in Figure 13.

In Table 9 the numerical values of drag on egg-shaped body in axial and transverse stagnation like quadratic flow have been calculated with respect to eccentricity $e$. It is found that drag values decreases from 1 to 0 in both axial and transverse stagnation flow situation as eccentricity $e$ increases from 0 to 1 . It is interesting to observe that in the limiting case as $e=1$, the case of slender spheroid or needle like body, drag comes out to be 0 . The variation of these drag values with respect to eccentricity $e$ are depicted in Figure 14 .

\section{Conclusion}

The authors have further developed the conjecture published in paper [1, 1999] by Datta and Srivastava and evaluated the general expressions of drag on axially symmetric bodies under the restrictions imposed in Section 2 placed in unbounded axial and transverse paraboloid as well as axial and transverse stagnation like paraboloid flows with appropriate choice of surface average velocity on body surface defined by Chwang and Wu [2, 3, 1975, parts 2 and 3]. These general expressions have been successfully applied not only to sphere and spheroid but also to egg-shaped body having left half semi-spherical and right half semi-spheroidal. The same may be applied to check on egg-shaped body when left half is spheroidal and right half is semi-spherical that the values of drag remains the same. The reason behind it is that the Stokes 
flow does not distinguish the fore and aft symmetry of the body. It may be easily analyzed that the proposed new analytical technique is very easy in comparison to the singularity method provided by other workers in the past for quadratic flow. Authors are searching the avenues of this analysis further for non-linear Stokes flow and other type of quadratic complex flows.

Acknowledgements. First author convey his sincere thanks to University Grants Commission, New Delhi, India, for providing financial assistance under major research project scheme[F.N. 39-55/2010(SR), 24-12-2010] at the Department of Mathematics, B.S.N.V. Post Graduate College, Lucknow(U.P.), India. Authors are also thankful to the authorities of B.S.N.V. Post Graduate College, Lucknow, to provide basic infrastructure facilities during the preparation of the paper.

\section{REFERENCES}

1. Datta, S. And Srivastava, D. K.: Stokes drag on axially symmetric bodies: a new approach, Proc. Indian Acad. Sci.(Math. Sci.), 109(4), (1999), 441-452.

2. Chwang, A. T. And Wu, T. Y.: Hydromechanics of low reynolds number flow. Part 2. Singularity method for stokes flows, J. Fluid Mech., 67(4), (1975), 787-815.

3. Chwang, A. T.: Hydromechanics of low Reynolds number flow. Part 3. Motion of a spheroidal particle in quadratic flows, J. Fluid Mech., 72, (1975), 17-34.

4. Stokes, G. G.: On the effect of internal friction of fluids on pendulums, Trans. Camb. Philos. Soc., 9, (1851), 8-106.

5. OBERBECK, A.: Über stationare flussigkeitsbewegungen mit beruksichtigung der inneren, Reibung. J. reine angew. Math., 81, (1876), 62-80.

6. Edwards, D.: Steady motion of a viscous liquid in which ellipsoid is constrained to rotate about a principal axis, Quart. J. Math., 26, (1982), 70-78.

7. Jeffery, G. B.: The motion of ellipsoidal particles in a viscous fluid, Proc. Roy. Soc. A. 102, (1922), 161-179.

8. TAYlor, G. I.: The motion of ellipsoidal particles in a viscous fluid, Proc. Roy. Soc. Lond., Ser. A, 103(720), April 03 (1923), 58-61.

9. Goldstein, S.: The forces on a solid body moving through viscous fluid, Proc. Roy. Soc. Lond., Ser. A, 123(791), March 06, (1929), 216-225.

10. Lighthill, M. J.: On the squirming motion of nearly spherical deformable bodies through liquids at very small Reynolds numbers, Comm. Pure Appl. Math., 5, 5, (1952), 109-118.

11. Hill, R. And Power, G.: Extremum principles for slow viscous flow and the approximate calculation of drag, Quart. J. Mech. Appl. Math., 9(3), (1956), 313-319.

12. Chwang, A. T. And Wu, T. Y.: Hydromechanics of low reynolds number flow. Part 1. Rotation of axisymmetric prolate bodies, J. Fluid Mech., 63(3), (1974), 607-622.

13. Chwang, A. T. And Wu, T. Y.: Hydromechanics of low reynolds number flow. Part 4. Translation of spheroids, J. Fluid Mech., 75(4), (1976), 677-689.

14. Johnson, R. E. And Wu, T. Y.: Hydromechanics of low Reynolds number flow, Part 5. Motion, J. Fluid Mech., 95(2), (1979), 263-277. 
15. Ho, B. P. AND LeaL, L. G.: Migration of rigid spheres in a two-dimensional unidirectional shear flow of a second-order fluid, J. Fluid Mech., 76, (1976), 783-799.

16. PARKer, G. W.: Projectile motion with air resistance quadratic in the speed, American J. Physics, 45(7), (1977), 606-610.

17. Majhi, S. N. And VASUdevaiah, M.: Flow separation in a viscous parabolic shear past a sphere, Acta Mech., 45, (1982), 233-249.

18. KAloni, P. N.: A note on the drag force on a spherical particle in a quadratic flow of a micropolar fluid, Int. J. Engg. Science, 21(8), (1983), 1001-1008.

19. Keh, Huan J. And Anderson, J. L.: Configurational statistics of Brownian dumbbells in a quadratic flow, J. Chem. Phys., 80, (1984), 1632-1639.

20. YuAn, FAN AND Wu WANG-YI: The Stokes flow of an arbitrary prolate axisymmetric body towards an infinite plane wall, Applied Mathematics and Mechanics, 8(1), (1987) $17-30$.

21. Yang, Seung-Man And Hong, Won-Hi: Motions of a porous particles in Stokes flow: Part 1. Unbounded single-fluid domain problem, Korean J. Chem. Eng., 5(1), (1988), 23-34.

22. Seki, Masako Sugihara: The motion of an ellipsoid in tube flow at low Reynolds number, J. Fluid Mech., 324, (1996), 287-308.

23. Haber, S. And Brenner, H.: Symbolic operator solutions of Laplace's and Stokes equations. Part II. Stokes flow past a rigid sphere, Chemical Engineering Communications, 27(5-6), (1984), 297-311.

24. HABER, S. AND BRenneR, H.: Hydrodynamic interactions of spherical particles in quadratic Stokes flows, Int. J. Multiphase Flow, vol. 25(6-7), (1999), 1009-1032.

25. Palaniappan, D. And Daripa, Prabir: Compound droplet in extensional and paraboloidal flows, Phys. Fluids, 12(10), (2000), 2377-2385.

26. Lin, W., Graham, A. L., Ingber, M. S., Abbott, J. R. and Leggoe, J. W.: Hydrodynamic interaction of two neutrally-buoyant smooth spheres suspended in plane Poiseuille flow: the BEM simulations versus the $M o R$ approximations, Computational Mechanics, 36(4), (2005), 307-319.

27. Pasol, L., Sellier, A. And Feuillebois, F.: A sphere in a second degree polynomial creeping flow parallel to a wall, Quart. J. Mech. Appl. Math., 59(4), (2006), 587-614.

28. Prakash, J., Rajasekhar, G. P. And Kohr, M.: Faxen's law for arbitrary Stokes flow past a porous sphere, Arch. Mech., 64(1), (2012), 41-63.

29. Srivastava, D. K., Yadav, R. R. And Yadav, S.: Steady Stokes flow past deformed sphere. Class of oblate axi-symmetric bodies, Int. J. Appl. Math. Mech., 8(9), (2012), $17-53$.

30. Happel, J. And Brenner, H.: Low Reynolds Number Hydrodynamics. Nijhoff, Dordrecht, The Nederlands, 1964, 1983.

31. Chan, P. C. H. And Leal, L. G.: A note on the motion of a spherical particle in a general quadratic flow of a second-order fluid, J. Fluid Mech., 82, (1977), 549-559. 